A Dissertation
presented to
the Faculty of the Graduate School
at the University of Missouri-Columbia
In Partial Fulfillment
Of the Requirements for the Degree
Doctor of Education
CHAUNCEY GRANGER
Dr. Paul Watkins, Dissertation Supervisor
December 2017


TRANSFORMATIONAL LEADERSHIP IN URBAN EDUCATIONAL SETTINGS

(C) Copyright by Chauncey Eldridge Granger 2017

All Rights Reserved 
The undersigned, appointed by the dean of the Graduate School, have examined the Dissertation entitled

\section{TRANSFORMATIONAL LEADERSHIP IN URBAN EDUCATIONAL SETTINGS}

presented by Chauncey Granger, a candidate for the degree of doctor of education, and hereby certify that, in their opinion, it is worthy of acceptance.

Dr. Paul Watkins

Dr. Charles Phillip Gause

Dr. Sharon E. Gunn

Dr. David L. Stader 


\section{DEDICATION}

The opportunity for a high quality education despite the challenges of poverty and the struggles of the inner city especially in the 1990's, would not have been possible without the many sacrifices made by people who invested in me. I will begin by acknowledging the sacrifices of time, energy, and the tireless work that my Mother, Sharon E. Johnson, dedicated to insuring that I received a rigorous and challenging educational framework to build upon before leaving for college and the venturing out into the real world. I will also acknowledge the sound advice and relative bible references that my grandmother, N. Purifoy, gave to me to keep me spiritually grounded and focused. I thank my Father, Major Granger Jr. for investing his time, advice and hard work ethic that he instilled in me. I thank my Brother, Jevon N. Granger, for keeping me grounded and always having my back in good and bad times. I thank my Sister, Sharronda Y. Williams for her calm demeanor and supportive attitude.

To my Wife, Deanna D. Granger, who has played a tremendous role in my professional growth as an educator by spending countless hours listening to my job related frustrations, celebrating my triumphs, and encouraging me during uncertain times. Deanna has also put her personal goals on the back burner in order to support the demanding schedule of my job, our children's schedules and brought balance to our family life during the dissertation process. I will always be indebted to her for the many sacrifices that she has made and endured over the years, along with her ongoing support for me.

Lastly, I would like to thank the many friends, colleagues, and teachers that have had a positive impact on my life. They have served as motivation to enhance my professional and 
personal growth as well as inspired me to become a lifelong learner. Thank you to Mrs. J. Smith Mrs. T. Morgan, Mrs. B. Dennis, Mrs. R. Brimm, Mr. Ron Woods, Mr. T. Brown Sr., Mrs. B. Collins, J. Ellis, C. Terry, Ms. V. Foggy, Mrs. E. Allen, Dr. N. Greer, Mrs. Jenkins, Ms. J. Jones, Ms. B. Issa, Mr. M. Taggert, Mr. and Mrs. F. Thompson, Mr. C. Purifoy, G. Alberter, B. Harriman, Dr. C. Helsel, Mr. C. McFarland, Dr. I. Buchanan, Mr. S. Jenkins, Mr. C. Levy, Mr. M. Tolliver, Dr. O. Headen, Mr. G. Pipkin, Mr. O. Issa, Dr. I. Clark-Jackson, Dr. L. Williams, East St. Louis Public Schools District 189, Chicago Public Schools, St. Louis Public Schools, Hazelwood School District to name a few. It is because of your influence that I have continued to persevere and strive to encourage and inspire others. My lifelong dedication will be to the families, children and educators that I have had the pleasure to interact with in the past and the foreseeable future. I can’t neglect to thank my children, Miric B. and Kennedy G. for your understanding, patience and for sharing our time with the many demands of my career. Rest in peace Blue, Lady and Winter, you will forever be loved and missed. 


\section{ACKNOWLEDGEMENTS}

It will begin by acknowledging and sincerely thanking the members of my dissertation committee: Dr. Paul Watkins, Dr. Charles Phillip Gause, Dr. Sharon E. Gunn, and Dr. David L. Stader, for sharing their expertise, time and commitment to excellence during this process. I wholeheartedly appreciate your feedback, experiences and suggestions in writing this dissertation.

I am forever grateful to my committee chairman, Dr. Paul Watkins, for the countless hours of preparation, for you and your wife inviting me into your home, for continuously supporting me during personal struggles that came along the way, for the immediate turnaround with feedback and suggestions, for the numerous and meaningful meetings at Starbucks, for your straightforward and honest opinions and most of all, for your belief in me. There were many times that I wanted to throw in the towel and you would send a text message to check in with me, an email giving me a gentle reminder of a deadline, or an honest, courageous conversation with me in person that kicked me in gear to continue and move forward despite the roadblock. You have served as an awesome chairman and a mentor and I truly thank you. 


\section{TABLE OF CONTENTS}

ACKNOWLEDGEMENTS...................................................................................................

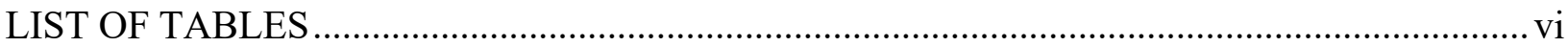

CHAPTER 1 BACKGROUND OF URBAN EDUCATION...................................................... 1

Problem Statement.............................................................................................................

Research Purpose

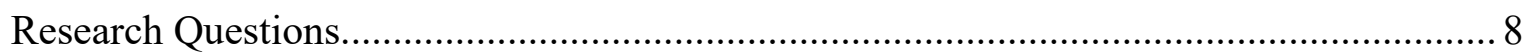

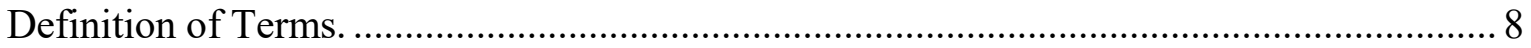

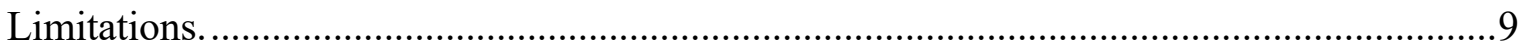

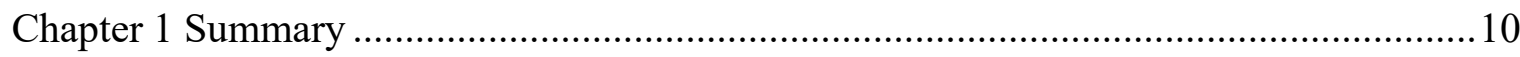

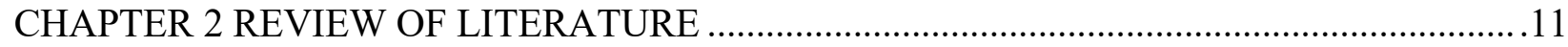

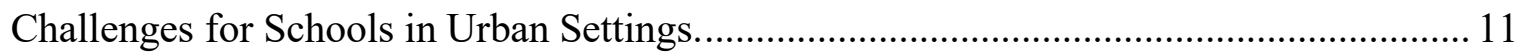

Urban Poverty vs. African American Family Structure...........................................12

The Circumstances of Poverty.............................................................................13

Attitudes Born from Deficit Thinking ................................................................ 14

Recruiting and Retaining Teachers in Urban Districts.............................................. 16

Leadership Staffing Challenges...................................................................................18

Building a Culture of Trust in Urban Schools .............................................................. 21

Building Trust in Leadership ..........................................................................2

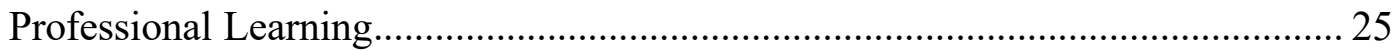

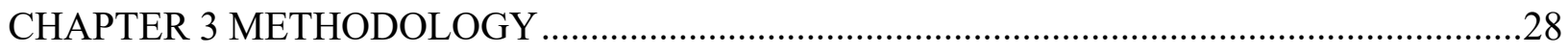

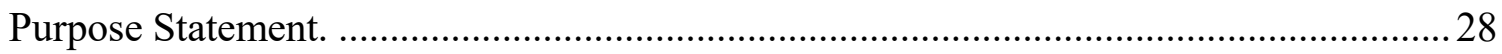

Research Questions ..................................................................................................2 29

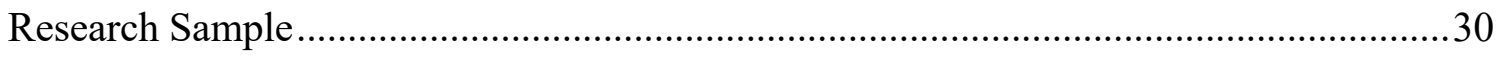

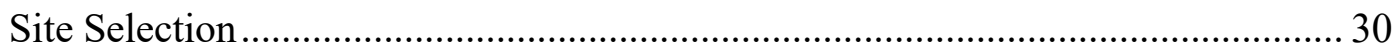

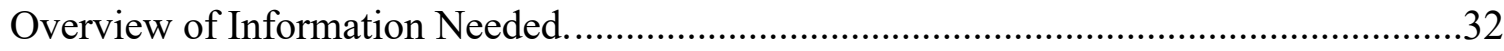

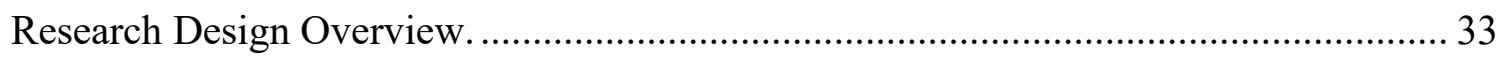

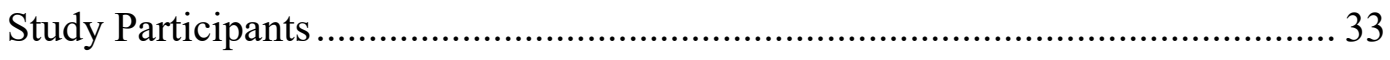

Format of Focus Group Sessions. ................................................................................. 34 


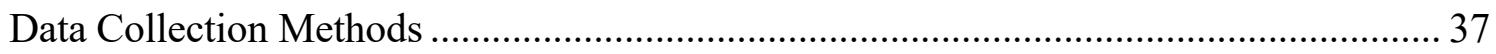

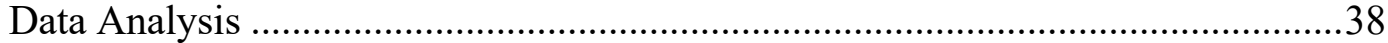

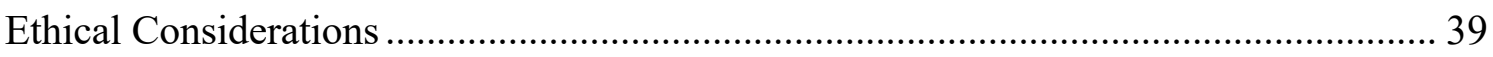

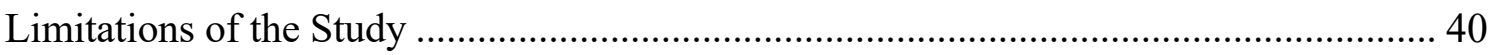

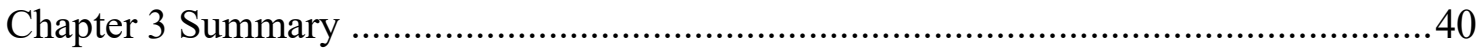

CHAPTER 4 RESEARCH, DATA COLLECTIONS AND FINDINGS.................................42

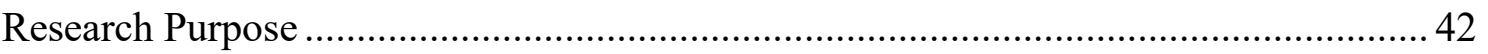

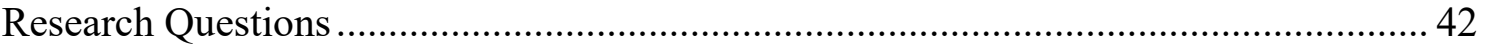

Research Methods and Data Collection Procedures .................................................... 43

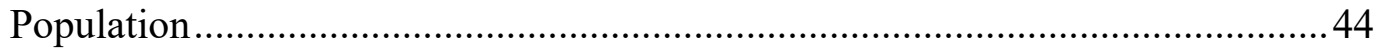

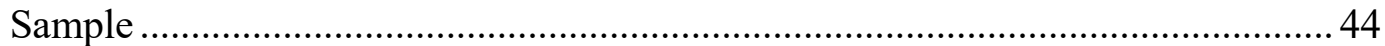

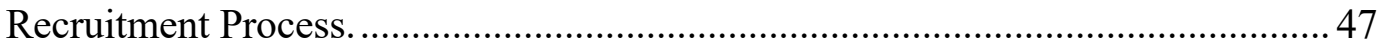

Focus Group Interview Process. ............................................................... 47

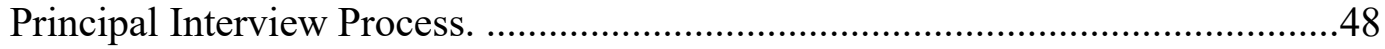

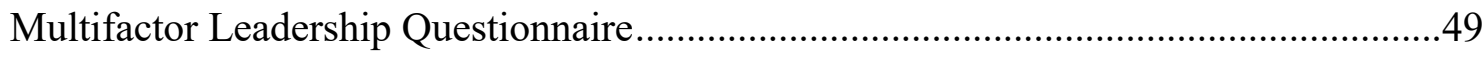

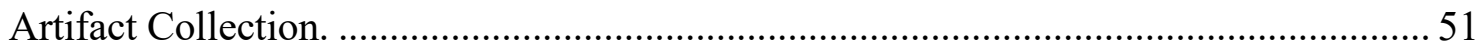

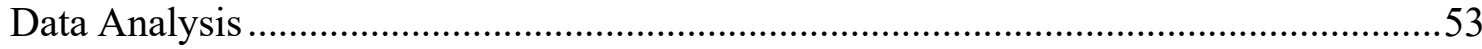

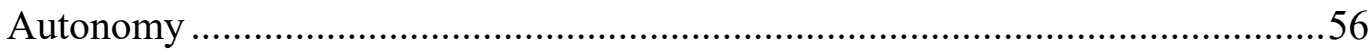

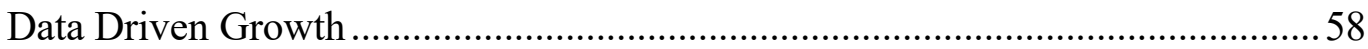

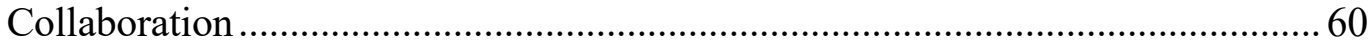

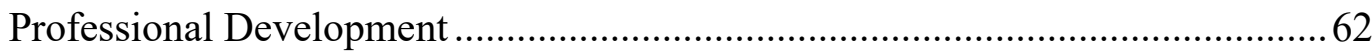

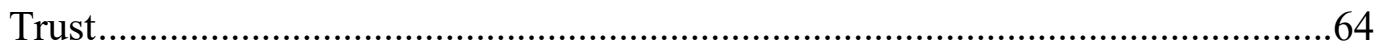

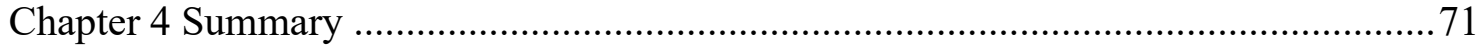

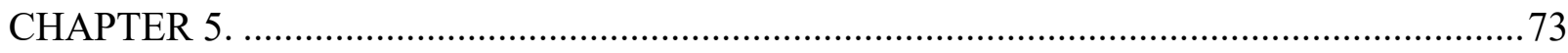

Research Findings, Conclusions and Recommendations............................................ 73

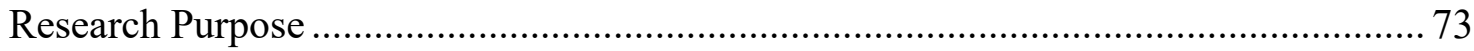

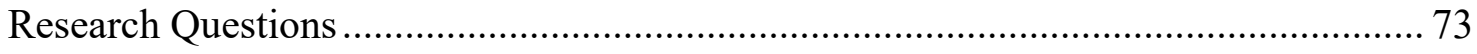

Research Methods and Data Collection Procedures. ..................................................74

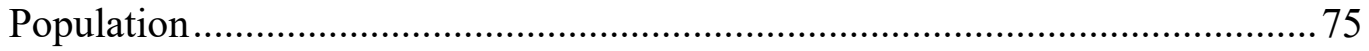


Sample

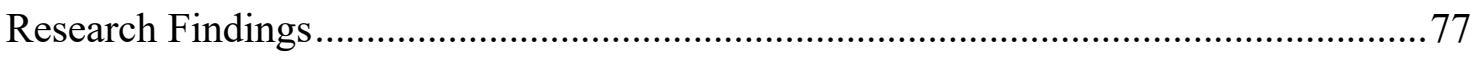

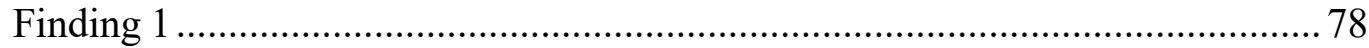

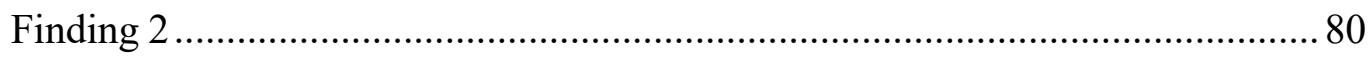

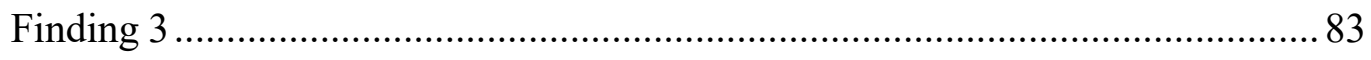

Unexpected Findings ................................................................................... 85

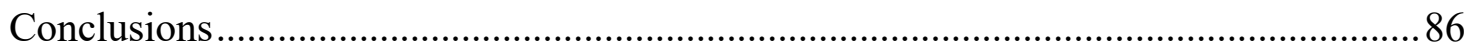

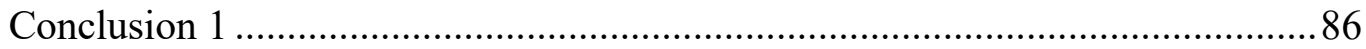

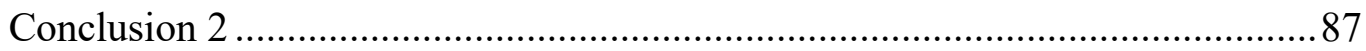

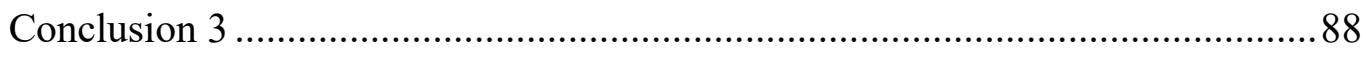

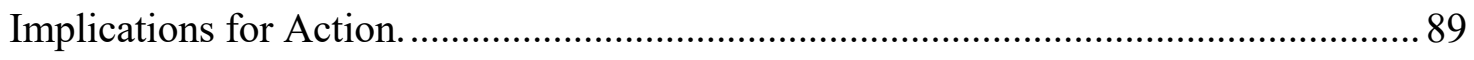

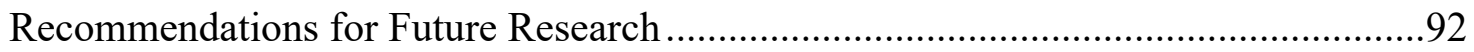

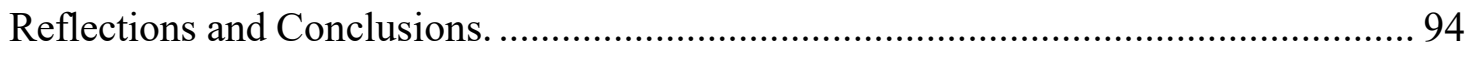

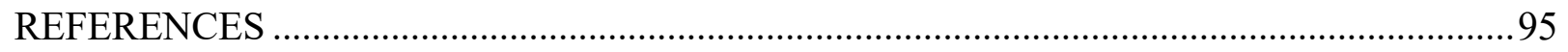

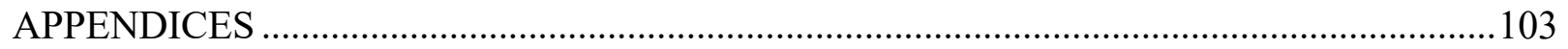

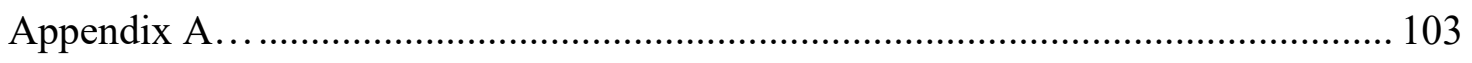

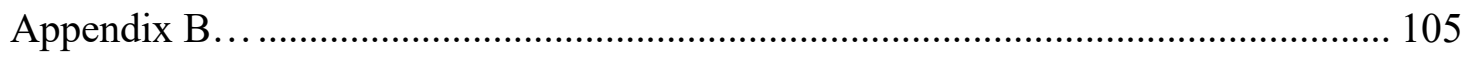

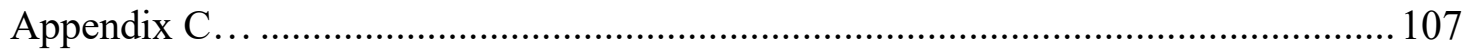

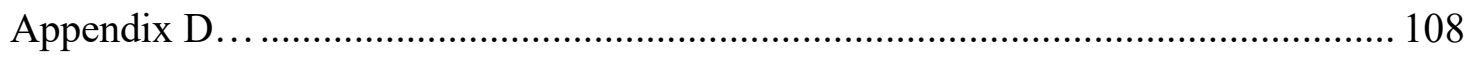

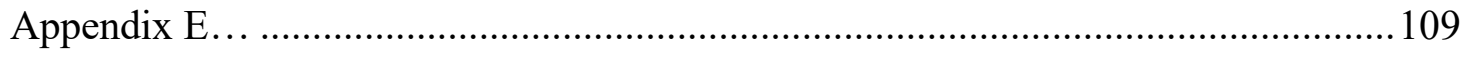

VITA 


\section{LIST OF TABLES}

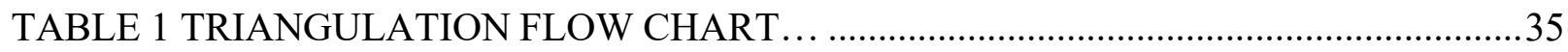

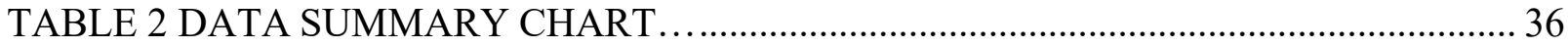

TABLE 3 ADMINISTRATIVE INTERVIEW DATA.................................................... 46

TABLE 4 FOCUS INTERVIEW DATA .......................................................................... 47

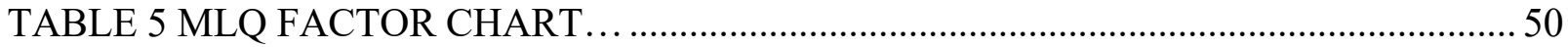

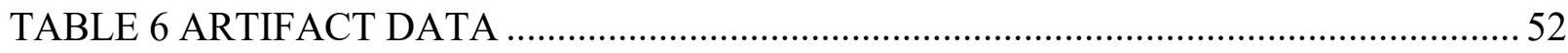

TABLE 7 FOCUS GROUP THEMATIC FREQUENCY CHART .........................................54

TABLE 8 THEMATIC FREQUENCY PRINCIPAL INTERVIEW CHART.......................66 


\section{CHAPTER 1}

\section{Background of Urban Education}

Urban education has both internal and external challenges that place inner city students at an extreme disadvantage to acquiring educational equity and access to knowledge and skills necessary to compete in a constantly evolving global society (Oakes, Franke, Quartz, Rogers, 2002). Jean Anyon (2005), identifies failed, short-term educational policies such as urban family dynamics, and underprepared teachers and principals as secondary contributors of the ills of urban education. Her position is an unpopular contrast to the mainstream theory and definition of urban education. She suggests that macroeconomic mandates such as minimum wage and housing policies as the root causes, thus having more global effects that eventually trickles down to affect the quality of urban education. According to her theory, urban education as we know it today is a mere consequence of ineffective economic policies which perpetuates conditions that yields a lack of access to essential services, equity, opportunity, social economic segregation and eventually poverty (Anyon, 2005).

Statistics cited from the (U.S. Department of Education, 1996), also showed disparities among low academic performance, socioeconomic status and the growing gap between Blacks and Hispanics and their white counterparts (Sigelman and Welch, 1991). Moreover, the projected increase in population for both racial groups only heightened the researchers' concerns and implied the need for change within the smaller structures of urban school districts at the school level.

Lyndsey Layton (2015), discovered that a majority of U.S. public school students are in poverty. Michael Rebell speaks to the disparity of financial success between socioeconomic groups widening despite the overall projected economic outlook of the country becoming more prosperous. These results warn of an increasing number of students entering public schools with 
academic challenges that put them at risk of not meeting the rigors of ever-changing state and national standards. This challenge is compounded by the majority of students living in poverty not having their basic needs met before they begin school each day. An even greater challenge are demands of high-stakes testing and pressure on urban educational systems and educators to meet the holistic needs of their children. At the same time, teachers and administrators must provide a high quality educational experience that will result in raising student achievement. They are also tasked to close the growing educational gap between urban students of color and their white counterparts in high performing school systems (Layton, 2015).

Moreover, globalization demonstrates the need for American society to begin to analyze and address the growing international educational gap between students in the United States and their foreign counterparts. Research trends show that American students are lagging in academic performance compared to students in Far Eastern countries like Taiwan and Japan, resulting in jobs being outsourced, increasing demand and competition (Tapia, 2013).

A downward spiral of student academic performance and the rise of international testing results place tremendous pressures on students, teachers, and principals to revamp the failing existing educational structures. Educators must rethink outdated teaching practices, increase higher order thinking strategies, realign local, state and national standards of learning, and keep pace with the rapid progression of technology as a means to meet the demands of a dynamic, global job market (National Center for Education Statistics, 2013).

High-stakes reform creates the arduous task for principals to orchestrate a paradigm shift in thinking for all members of the organization. Reshaping school paradigms require healthy but critical feedback to be provided from leadership to guide members of the organization to become reflective learners, willing to analyze decision making, root causes and unintentional outcomes. 
The transformational approach creates moments of discomforts that need to be carefully managed in order to yield positive outcomes (D. Anderson \& Anderson, 2011). It will require transformational leaders to communicate a clear vision for the school and to sustain that vision of success on a long-term basis. Transformational leaders are described as leaders who inspire and motivate members of the organization to high levels by intertwining the member's personal goals with the organization's goals. Motivation increases loyalty, trust, buy-in and respect for the leader. Leader inspired motivation also fulfills the member's personal needs and the organization's needs of productivity as a transactional exchange (Tipu, S.A., 2012). Leader inspired motivation eventually leads members of the organization to take initiative in problem solving and to produce positive and profitable outcomes for both parties (Tipu, S.A., 2012).

\section{Problem Statement}

One of the major challenges in urban education lies within school leadership. In many schools teachers feel isolated from colleagues, school leadership and the community. As a result, they withdraw and become more compliant to completing paperwork rather than being committed to the overall success of their students.

Particularly in urban secondary schools, principals fail to facilitate opportunities for teachers to meet collaboratively as a method to approach difficult decision making relative to teaching and learning. This lack of opportunities for collaborative planning is exemplified through neglecting to allocate specific time in the academic schedule for teachers to meet daily and or weekly with content teams and grade level teams. The absence of collaborative time denies teachers opportunities to create common lesson plans, meet as data teams to discuss academic achievement trends, best practice sharing, conduct collegial classroom walkthroughs, grade level team meetings, behavioral intervention meetings, joint parent meetings, professional 
learning communities, and mentor meetings to name a few. As a repercussion, teachers are driven by cynicism and frustration (Fullan, 2008).

Inner city secondary schools are particularly vulnerable to isolated departmental level structures that often separate teachers from the rest of the school and as a consequence, yields a by-product of a poorly managed infrastructure. This structure may encourage collaboration within the department but that collaboration does not extend to the general mission of that school (Andy Hargreaves as cited in Leithwood \& Jantzi, 1990). With the absence of a schoolwide culture for excellence the "superglue", critical to joining people to meaningful school outcomes, will not occur (Bolman \& Deal, 2010).

Chubb and Moe argue that radical structural changes are essential to address the challenges of urban education. They believe that short term solutions such as state takeovers, school vouchers and privatization only add to the problems of low performing schools. However, the same data from which Chubb and Moe based their argument on also suggests that there are a number of schools that are showing significant improvement on national standardized tests (Chubb and Moe, 1991). School districts that are showing improvement are largely located in suburban areas. Those that fall short are primarily located in large urban areas with the majority of students being African American and Hispanic.

Building trust with the staff, community, and students is seemingly an insurmountable task for principals in urban schools. Trust between leadership and faculty is a key component to effective communication of concerns, goals, needs and high levels of collegiality. However, collegiality does not come to leadership automatically. It is cultivated on an ongoing basis through continuous respect for the ideas of others, engaging faculty in meaningful discussion, and assuring that all opinions are shared (Fullan 2003). Holding high expectations communicates trust in the competence in faculty. Maturing competence emerges from 
professional learning opportunities. As Fullan tells us, leaders must be cognizant of building trust to sustain a committed culture. Organizational changes have to become a part of the daily operations, routines and rituals (Bolman \& Deal 2008). It will eventually come to a point where the words of the school vision and mission are living and breathing practices that can be observed at any given time. If not, the culture is subject to significant losses (DuFour and Eaker 2010).

Examples of open communication channels would be leadership teams that consist of a variety of different faculty members that would resemble the faculty at large. There would be established norms and processes to follow during each meeting. Problem solving protocols help to keep topics on course as well as techniques implemented as a means to hear and respect all voices. An agenda shared prior to meetings tends to promote transparency. In addition, minutes from meetings should be openly shared with the staff at large with an action plan and next steps. Such processes allow an open flow of communication of ideas to become a norm and begins the process of trust building with team members and staff.

Transformational leaders encourage risk taking as an accepted part of the organizational culture. Transformational leaders are innovative thinkers that plan with the end in mind, predict unintended consequences of decision making and empowers employees to gain relevant experiences that are both aligned to their personal goals and the overarching goals of the organization (Bolman \& Deal, 2010). Risk taking and out of the box thinking are encouraged as a means to break continued cycles of flat lined results. Transformational leaders attract various talents to the organization that will add a variety of perspective and character to the think tank. Innovative people and fresh ideas will enhance the opportunity for continuous collaboration and innovation to emerge from the collective knowledge of the organization (D. Anderson \& Anderson, 2010). 
In addition to the aforementioned challenges that face urban educators, in the last twenty years, the role of the principal has drastically evolved from a managerial role to an instructional leader role (Dhuey \& Smith, 2014). Principals are charged to manage an array of resources, ensure the safety of students and staff, as well as meet the academic needs of all learners. The new mandate of the Every Student Succeeds Act pushes the academic expectations to much higher standards of learning than ever before (National Education Association, 2008).

This requires principals to become change agents that will challenge teachers to explore new and innovative ways of approaching teaching. This is a paradigm shift with the emphasis changing from teaching to learning. Instructional leaders are now encouraged to push students to new and unchartered waters of student led experiences in classrooms (Bush, T., 2009).

The roles of students are evolving to a point where they are now asked to lead discussions, share their thought processes that led to the answer instead of the mere final answer without an explanation of its derivation. The roles of teachers are evolving from dominating the conversations and dictating the information, to facilitating the educational experiences and stepping back and allowing students to take more ownership in activities and discussions. This can be a very uncomfortable process for all stakeholders with the accountability of its success primarily falling on the shoulders of the principal (Bush, T., 2009). This can be especially challenging for principals in urban settings.

Boyd, Grossman, Lankford, Loeb and Wyckoff, (2011) described urban schools as having a majority of nonwhite, poor, and low-performing students who are taught by teachers that either are non-certified for the areas that they are currently teaching, or have failed the basic competency tests and only have provisional certificates. Lankford, et al. (2011), went on to explain how teachers in urban schools are more likely to either transfer or quit and seek employment in suburban districts with white, non-poor students that pay an average of 10,000 
dollars more for beginning teacher salaries.

Consequently, the teachers that are more likely to leave the urban school districts are more likely to have more skills than the teachers that stay (Boyd, Lankford, Loeb and Wyckoff, 2005). The departure of highly qualified teachers may increase or worsen the disparities of inequitable distributions of highly qualified and certified teachers. The article generalizes the skills of the teachers that are "left", assuming that they wanted to leave, as poor or subpar. However, there is a flight or exodus of young teachers that gain their initial experience in urban districts eventually moving to more affluent suburban districts. This article clearly defines urban districts and explains the challenges facing urban education with the lack of high quality teacher retention and subsequent high staff turnover.

\section{Research Purpose}

The purpose of this study is to examine leaders and faculty of secondary urban schools that provide a culture of commitment to high levels of collegiality, high expectations, and professional learning. This research will illustrate how leaders in urban, secondary schools transform the cultures of schools and raise the commitment levels of teachers while sustaining academic gains for students. This research will search for evidence of a school environment conducive to the real world challenges of the twenty first century urban education. Lastly, this research will also investigate the promotion of professional learning and it becoming a viable part of the culture. Below are the three essential research questions that will guide this investigation. 


\section{Research Questions}

The research questions guiding this study are:

1. How do leaders of urban secondary schools with sustained culture of high academic performance develop collegial trust?

2. How do school leaders of urban secondary schools demonstrate academic expectations for themselves and their students?

3. How do school leaders of urban secondary schools promote a professional learning culture?

\section{Definition of Terms}

Culture. The way in which an organization operates as evidenced by actions observed on a consistent basis. The shared beliefs, values, vision, and mission of an organization (Lindsey, Jungwirth, Pahl, 2009)

Globalization. For the purpose of this study, globalization is the phenomena of international competition in education, business, trade, and ideas in which the exchange of communications in these areas were made readily available and accessible to people all over the world as a product of ongoing technological advancements (Ezaki, 2015).

Urban Education. For the purpose of this study, urban education can be defined as education in an inner city setting. This would in most cases include the effects and challenges of poverty, which is a hindrance to student achievement (Boyd, Grossman, Lankford, Loeb and Wyckoff, 2011).

Professional Learning Community. For the purpose of this study, PLCs are communities of professionals learning collaboratively in an ongoing, inquiry based, analytical 
approach for continuous improvement. Information is shared through best practices, data driven practices and a respect for differences within community (Lindsey, Jungwirth, Pahl, 2009).

Transformational Leadership. For the purpose of this study, the transformational leadership style encourages risk taking as an accepted part of the organizational culture. Transformational leaders are innovative thinkers that plan with the end in mind, predict unintended consequences of decision making and empowers employees to gain relevant experiences that are both aligned to their personal goals and the overarching goals of the organization (Bolman \& Deal, 2010).

Every Student Succeeds Act. For the purpose of this study the ESSA is a law passed by Congress in 2015 that established the government's role in public educational funding, monitored through annual standardized testing for students from 3rd to 8th grades (United States Department of Education, 2015).

\section{Limitations}

Some potential limitations to the study are time, group dynamics, and cost. According to Krueger, the time invested in focus groups could exceed 276 hours (Krueger, 2014). This time includes the planning and creating the questioning, selecting participants that are the right fit for the study, managing the meeting and insuring that the communicational flow remains steady and the group stays focused. The last piece that can be very time consuming is the analysis which includes transcribing the notes and discussions in the interview, coding and selecting themes and preparing the written report. This limitation is something to consider before embarking on such a rigorous style of qualitative research.

Group dynamics may create further limitations to the study. Group discussions are vulnerable to one or two individuals dominating the entire interview. In focus groups the purpose 
is to gather various perspectives and insights on a particular matter. In order for this to be possible, a skilled moderator must insure that all voices are heard and the group stays on task. Groups also tend to sway the opinions of others or "group think". This occurs when individuals begin to conform to the thoughts and opinions of the group despite philosophical differences that go against their personal beliefs (Kruger 2003). Consequently, the moderator has to insure that the conversation is interactive and everyone feeds off each other's' responses and give more verbal cues than nonverbal so that it will not take away from the meaning or thought by it being audio-recorded and not videotaped.

The last limitation involves cost. If a researcher is not careful, the costs could exceed the confines of a modest budget. Kruegar explains that the costs included in focus groups include travel, equipment, supplies, food, gifts and other miscellaneous items (Kruegar 2012). Financial responsibilities can definitely limit the resources and impact of the study on the broader community. The limitations mentioned should be carefully monitored and anticipated when using the focus group approach to research.

\section{Chapter 1 Summary}

I am interested in studying how school leaders in urban schools create a culture that guides teachers and students to raise expectation levels and to become committed to shared goals that lead to academic achievement. It is my perception that there is a significant potential for this study to affect educational leadership decision making in underperforming, urban, secondary, school districts. This research will contribute information to urban school leaders that are in the beginning stages of building staff capacity and creating a collaborative culture of committed educators. 


\section{CHAPTER 2}

\section{Review of the Literature}

With the national mandate, Every Student Succeeds Act and high stakes testing, there is a heightened sense of urgency in underperforming, urban schools to ensure that all students are proficient in Math, Science, and English. The emphasis in urban education is for principals to become instructional leaders rather than managers. However, the pressure in urban education is for principals to raise student achievement in the aforementioned areas.

In this study, transformational leadership is examined through the lens of school leadership in an urban setting. The literature begins with the challenges that principals face in urban schools. Once the challenges are explained, the complexities of building a culture of trust in urban schools are explored. These challenges transitions seamlessly into the process of implementing professional learning communities in urban, school settings.

\section{Challenges for Schools in Urban Settings}

One of the most prevalent challenges that urban educators face is urban poverty. Urban poverty can be described as the catalyst of the multidimensional challenges that urban educators face head on every day (Bates, 1991). Although most poverty studies conclude that Caucasians account for the vast majority of families stricken with the effects of poverty, there is an enormous disparity between racial dispensations of high crime ridden neighborhoods, teenage pregnancies, section eight housing, welfare dependency, and mortality rates of males, to name a few (Wilson, 1985). Wilson's research conveys the effects that urban poverty inflicts on the black lower class's plight towards equality in education and society as a whole (Wilson, 1985). 


\section{Urban Poverty vs. African American Family Structure}

Wilson's research further implicates the derivative effects that urban poverty has on the family structure of African Americans. Wilson cited Daniel Patrick Moynihan's (Wilson, 1985) report on the Negro family. Moynihan was cited as stating the central problems for the black lower class were divorce rates, out of wedlock births, welfare dependency, and female heads of households. Moynihan gave a historical account of poverty and its origins stemming from slavery and continued with the discriminatory practices of American society (Moynihan, 1965).

Moynihan suggested that female-headed households were significant data points that indicated sources of poverty cycles in African American families. These families were assembled by the joblessness of African American males, high incarceration rates, divorce rates, illegitimate children, and a number of other contributing factors. The (United States Bureau of the Census 1983) validated Moynihan's claims by showing statistical results that $81 \%$ of all African American female-headed families lived in urban areas. $77 \%$ of those were in inner-city residential areas. 57\% of inner-city African American female-headed families were classified as living in poverty due to only having one source of income (United States Bureau of the Census, 1983). According to (Bates, 1991), 80\% of children born to unmarried teenagers live in poverty. In comparison to 2014 statistics, $37 \%$ of Black families headed by single women live below the poverty line. The highest poverty rates (46\%) are for Black families with children which are headed by single Black women. This data is significant, considering more than half (55\%) of all Black families with children are headed by single women (United States Bureau of the Census, 2015). Wilson confirms Moynihan's theory by describing the continued eight out of ten year cycle of poverty that is perpetuated by families over the years as "intergenerational transmission of poverty". However, Wilson noted that the most significant difference between female headed families and married couples was income (Wilson, 1985). 


\section{The Circumstances of Poverty}

The cycle of poverty has continued to be perpetuated from Moynihan's research period through the 1990s and into the 2000s. Wilson labeled this group as "a growing black underclass", in essence trapped by the circumstances of poverty. The circumstances of poverty contributes to the challenges in urban education's lack of parental involvement, student's basic health needs not met, which may lead to special educational modifications, and accommodations for students of low income (Wilson 2011). External distractions are also circumstances of poverty affecting school culture and climate such as neighborhood crime, behavioral disruptions in classrooms, and a lack of financial capital. The aforementioned distractions are significant symptoms that require a focus on equity from state and federal resources for urban districts. By addressing the external distractions, it will begin the process of closing the widening achievement gap (Wilson, 2011). In 2009, the achievement gaps between African American boys and White boys were 27 percentage points in reading and 39 percentage points in math (Wilson, 2011).

Bates (1991) characterized a set of poor people as those truly living a substandard lifestyle and are considered at risk because of extreme circumstances of poverty. This at-risk group has experienced intergenerational transmission of poverty through the course of time, long-standing unemployment, high crime rates, and health issues that plague families because of the lack of access afforded to middle class income families. Socially, this group is out casted and left with a helpless feeling of powerlessness and desperation (Bates, 1991).

The feeling of powerlessness leaves a lasting effect on our most vulnerable citizens, children. Poverty multiplies the likelihood of negative experiences for children at an alarming rate (Danziger and Stern, 1990). Poverty increases the mortality rates for children exponentially. It almost guarantees that the average child will experience a devastating life event such as 
murder of a loved one, witness or victimization of a violent crime, ongoing socio-emotional issues, developmental delays in growth both physically and mentally and the susceptibility to prison (Danziger and Stern, 1990). Children of poverty experience many obstacles both within and outside their communities. One such seemingly insurmountable obstacle is deficit attitudes.

\section{Attitudes Born from Deficit Thinking}

There are many teachers who believe that poverty is a consequence of an absence of willpower, a deficiency of intrinsic motivation and a sign of inferiority (Battey \& Megan, 2016). Research on urban education commonly concludes that teachers bring deficit attitudes with them due to the scarcity of cultural exposure and blame for the shortcomings of children and families that live in poverty (Battey \& Megan, 2016). Some examples of deficit attitudes include lowered expectations for learning capacity, stereotypical mindsets and low standards of social behavior. These deficit attitudes pervade in affluent communities, schools, the media, entertainment, music and the American culture. These outlets serve as constant reminders and negative stimuli that can cause poor children to feel disconnected, disenfranchised and demoralized (Danziger and Stern, 1990). Deficit attitudes disconnects the poor from the prevailing culture at large. Poor families feel disenfranchised from opportunities to thrive in society and demoralized mentally and socially (Danziger and Stern, 1990).

Deficit attitudes hinder teachers from connecting with students and families and contributes to the detachment between school and home. Deficit attitudes have negative effects on the culture and climate of the school which ultimately leads to most urban schools labeled as hard to staff with highly qualified teachers. Although many efforts attempt to address issues of poverty through professional development, the idea of engaging in courageous conversations about race is usually not received well by some teachers (Battey \& Megan, 2016). This disconnect is due to ideas being more subtle and indirect. Some teachers are not aware when they 
make comments through narratives that share their experiences teaching in urban, inner city areas. Moreover, teachers avoid conversations about race and are non-participatory during professional development opportunities (Battey \& Megan, 2016). Many urban school districts are making it mandatory for employees to participate in cultural competency trainings as an effort to combat deficit attitudes and misconceptions, repair and restore broken relationships between home and school and ultimately, to help close the achievement gap between African Americans and other minorities including their white counterparts (Finn \& Johnson 2005). However, few teachers are open to the ideas and the reflective thinking processes entailed by cultural competency trainings.

Fortunately, cultural-competency training helps combat the common assumption that African American and Latino students are incapable of learning mathematical concepts instead of accepting help for their inability to adapt and implement effective strategies that will support struggling students (Battey \& Megan, 2016). Lowered expectations are more examples of covert ideas toward students in poverty stricken areas. Teachers are not pushing students to higher levels of achievement based on the notion of stereotypes inferring that students are not ready to move to higher order thinking skills necessary to progression into advanced classes in urban schools because of the lack of foundational skills (Finn \& Johnson, 2005).

On average, students spend significantly more time with teachers than they do with family (Wentzel, 2009). In urban schools, teachers are charged with cultivating meaningful relationships with students and families as they are a crucial influence on student's social, mental and academic development. Relationship building will eventually assist students to feel connected and a part of the school culture (Wentzel, 2009). Teachers should be cognizant of their presumptions and beliefs about students and families that live in poverty so that they don't add to the negative stereotypes that discourages families to let their inherent, protective guards down 
and begin to trust educators as people who care about their children. Finding effective educators to serve this vulnerable population are increasingly hard to find (Jacob, 2007).

\section{Recruiting and Retaining Teachers in Urban Districts}

Staffing urban schools with highly qualified teachers is an arduous task for leadership especially in the core subject areas of Math, Science and English Language Arts. Staffing urban schools located in impoverished areas are even more challenging in comparison to rural and suburban areas (Jacob, 2007). According to Jacob (2007), urban teachers have less experience, certifications and credentials than their suburban equivalents. The lack of highly qualified and certified teachers in urban districts can be attributed to urban teacher shortages. The increasing demand for teachers in urban school districts can be attributed to early retirements, low student attendance and enrollment but the main cause for the shortage is turnover.

Teacher migration away from inner city schools creates a considerable amount of the turnover experienced in urban school districts (Olsen \& Anderson 2007). Highly qualified teachers are lured to suburban and private schools by more competitive wages, better facilities and other incentives that are exceedingly attractive to prospective candidates (Olsen \& Anderson, 2007). Teachers are following a "migration pattern" towards affluent, suburban areas. Ingersoll adds that teachers in high-poverty areas are 50\% more likely to move than those in less poverty stricken schools (Ingersoll, 2003). Consequently, this results in principals left with no option but to staff the remaining vacancies with noncertified teachers (Olsen \& Anderson 2007).

Ingersoll (2004), noted several root causes for teachers to leave urban school districts. One reason for leaving is inadequate support from the leadership. Inadequate support includes a lack of feedback and follow up in respect to evaluative or observation feedback. Teachers become very frustrated when principals walk into their classrooms, stay for a few minutes, fill out a form and leave the room. Teachers enjoy praise, acknowledgement and descriptive 
feedback. Another root cause for teachers to leave is unprotected instructional time. Teachers become increasingly dissatisfied with a high number of disruptions including announcements via loudspeaker, unresolved disciplinary issues, subbing during planning periods, countless amounts of paperwork, and the added pressures of high stake testing (Freedman \& Appleman, 2009).

Limited opportunities for collaboration and collegial work can cause a feeling of being disconnected or isolation for teachers (Payzant, 2011). When time is not embedded within the academic schedule, teachers can become isolated from other teaching perspectives, strategies and best practices that could eventually result in frustration, cause stagnation and end in burnout. Teachers new to the profession are especially at risk when not supported and nurtured. Insufficient support in the first few years of teaching usually results in turnover over 30 percent of the time in urban schools (Payzant, 2011).

Urban schools also have high numbers of poor children in comparison to rural and suburban districts. In fact, the National Center for Education Survey indicates 56 percent of students receive free and reduced lunches 40 percent are classified as Title 1 students versus 32 and 20 percent in suburban districts (NCES 2003-2004). Urban poverty brings with it the unattractive elements of high rates of unemployment, violent crimes and the absence of social capital or positive role models. Aside state and federal funds, urban districts must contend with depleted tax bases, which puts a strong reliance on state and federal funds (Jacob, 2007). Depleted tax bases in most cases yields elevated mobility rates for students. Schools are becoming revolving doors for students that move in and out of the district occasionally several times per school year. Elevated mobility rates becomes disruptive to the climate of the school and the dynamics of the classroom, affecting the learning environment and eventually student achievement (Jacob, 2007). A combination of teacher defections and urban poverty present many challenges to inner city school leaders. 


\section{Leadership Staffing Challenges}

Maintaining a fully certified staff is another ongoing challenge for principals in urban school districts. Principals are tasked with recruiting highly qualified and certified teachers, competing with private and suburban schools with the promise of better wages and working conditions, and more efficient hiring practices of competitive districts.

Missouri school districts (not unlike other districts throughout the nation), are penalized for not hiring certified staff members in schools (Department of Elementary and Secondary Education, 2016). Title 1 schools, which are usually urban schools with high poverty rates, are especially vulnerable to high turnover and student transiency. In fact, if the principal does not hire a certified teacher, parents are notified to inform them that their child's teacher is not highly qualified. Such a policy can cause an array of problems for the principal, from upset parents to disappointed students (Ingersoll, 2014).

Principals not only have to fill vacant positions, they must be prepared to make adjustments with the constantly looming possibility that other teachers in their buildings will be enticed by competing districts searching for experienced, urban teachers to fill openings in suburban and private schools. Urban teachers are less inclined to remain in the same school for more than a few years (Jacob, 2007). Not only does recruiting and hiring make a difficult summer of recruiting for a principal, it has potentially serious consequences. One limitation of highly qualified teacher recruitment include the lasting ramifications that high mobility teachers have on a school culture. In addition, losing qualified staff requires rebuilding the capacity of new staff, braking long lasting relationships with school families, and the pressure of fully staffing classrooms before the opening can complicate and overwhelm an urban school leader.

Therefore, if urban principals fail to staff buildings before the first day of school there are few options left. Option one is for the principal to hire an inexperienced teacher, fresh out of 
college with no apparent conceptualization of the demands of teaching in an urban school. Second, the principal can choose to hire an educator with a temporary authorized certification which expires after one year. Third, the principal has the option to hire a long term substitute as a temporary solution to the problem. Fourth, the principal can choose to increase class sizes of the existing teachers until a qualified teacher is hired or hire a substitute with a provisional or temporary certification (Department of Elementary and Secondary Education 2016). These are not popular choices among the teaching staff or parents. Lastly, once the principal runs out of options, he or she is mandated to freeze the hiring process per the human resource department because they reserve the right to randomly move teachers from other buildings to fill the vacancy (Jacob, 2007).

Due to larger numbers of students and the amount of bureaucratic oversight involved in the hiring process in urban districts, principals are at a disadvantage when it comes to smaller suburban and private schools hiring processes. Smaller districts usually have more efficient systems in place in respects to hiring staff (Jacob, 2007). However, larger districts often possess more capabilities to host large scale recruiting fairs than their smaller counterparts. In larger school districts there are more organized Unions. This can complicate the process and diminish the power and influence of high ranking district officials.

Urban districts benefit from alternative certification programs with the number of teachers staying in urban, high poverty, hard to staff schools. The lure for these teachers has been career advancement opportunities and the advantage of connecting theory to practice in the experiences gleaned through this unique exposure to real situations (Freedman \& Appleman, 2009). Teachers feel more prepared and have a deep understanding of the challenges of urban education. Teachers also feel that they are fulfilling their intrinsic and moral obligation to serve and help students from poverty stricken communities (Freedman \& Appleman, 2009). Overall, 
the goals of the school are in alignment with the personal goals of the teachers educated in the alternative certification pathway as opposed to the traditional student teacher experience (Freedman \& Appleman, 2009).

Saint Louis Public Schools, like many urban districts, are at a disadvantage because in most cases, principals are not able to begin the hiring process until late May or early June (St. Louis public schools Article R4256). Most of the highly qualified teachers have already been hired and have begun summer professional development or summer school classes. This presents a complication for urban principals because they are now pitted against other urban districts to attract and hire the best candidates from the leftover pool of candidates already picked over by suburban and private school districts. On numerous occasions, under performing school leaders are competing within the same districts for the remaining talent in district hiring databases (Jacob, 2007).

Hiring practices need to be continuously examined in an effort to refine the search processes and to increase efficiency and the quality of talent hired in urban districts. The aforementioned challenges for leaders in urban districts often leads to a process of passing along ineffective teachers to understaffed, low performing schools. Thus serving as dumping grounds for high performing schools and districts.

As a result of high turnover of teachers in urban schools, alternative certification programs provide both theoretical and practical experiences for up and coming teachers as a proactive measure to provide support and decrease the trend of turnover in schools. Some school district's alternative programs incentivize participants by forgoing tuition, stipends, loan forgiveness and many other monetary opportunities for participants. However, the stipulations with the various perks of the programs commonly require participants to commit to teaching in respective districts for at least 5 years. This out-of-the-box thinking has led to retirement 
incentives as well to encourage educators to become vested into the school district, community and the lives of students living in impoverished conditions (Freedman \& Appleman, 2009).

A principal fraught with the many urban challenges outlined above must also set and manage the tone of a building. The principal, together with staff, is responsible for creating a positive and nurturing culture in the building.

\section{Building a Culture of Trust in Urban Schools}

The culture of school establishes the tempo for what is to happen next regarding student outcomes. The characteristics of the culture are the norms set by the staff by establishing the mission and vision statements. These are the core values and beliefs that the organization's purpose for existence is based upon. Core values are the non-negotiables that dictate how the school will prioritize its major foci and what the school will be known for (Bates, 1991). Building supportive relationships with children, teachers, school, home and the community at large will make the difference in establishing a culture conducive to teaching and learning (Comer, 1988). Relationships will also minimize barriers between school and external stakeholders. Smaller schools with fewer staff serving fewer neighborhoods can help impact stronger relationships with key stakeholders (Raywid, 1994).

A small schools movement represents the downsizing of schools, offering an intervention towards closing the achievement gap and a means to address disconnected relationships in larger urban schools (Raywid, 1994; Carter and Keiler, 2009). The movement ultimately creates a culture of trust between faculty and the school leadership (Dorman, 2012). Small schools affirm personal relationships through smaller class sizes as a means to create community within the classroom and to facilitate opportunities to build trusting and strong relationships. Within this model, small advisory groups are created to foster and develop personal bonds between teachers and students. The advisory class is maintained as a small manageable size so that the approach is 
personable. The groups are looped yearly through the duration of the student's experience at the school. Looping helps to keep the relationships intact and the communication with families consistent from year to year as well as insuring a high rate of student-teacher-parent interaction. The overall theory is that by maintaining the advisory groups from year to year, teachers will create tight bonds with the students and families (Dorman, 2012). Tighter bonds with families will yield a deep understanding of the strengths and struggles of the students as learners. Teachers will better understand home life situations and how they impact school, special interests of students and a true snapshot of the longitudinal progression of students developmentally, academically, and socially over time. This holistic approach creates a bond of trust between parents, staff and administration as well as school and home (Dorman, 2012). Dorman (2012), added the importance for urban educators to understand that students will share emotional aspects of their lives that may be disturbing and difficult to process. Teachers must then be able to actively listen and accommodate students. Accommodation includes various outlets for students to write, illustrate and express themselves and their current emotional state and connect those experiences with the curriculum for relevance. Teachers review and respond to students. Teacher use the findings from communications to inform next steps of recommended support whether it be to a counselor, social worker, school psychologists, or the department of family services. The intended outcome is for teachers to move from sympathy to empathy while maintaining a high level of expectations in the classroom (Gay, 2000). It is also important for teachers to show the humanistic side of themselves and share personal moments of their lives as another way to connect with students. Time and effort is another method of demonstrating care and fostering trust. Showing compassion is crucial to students that live in poverty and lack social capital. Through the acts of sharing personal time with students and attending extracurricular activities and home visits, relationships become 
stronger and trust is built. Once the trust is built, students are intrinsically motivated to impress their teachers through academic performance. Students are also prone to share information that could have drastic impacts on their lives or the classroom and school environment. Teachers are also able to impart their content knowledge upon students and their passion for education is renewed.

The small schools initiative is a productive prototype of how to build trust with families in urban schools through extensive relationship building and investing personal time with students outside the regular school day (Raywid, 1994). Investing personal time requires teachers to go above and beyond the contractual obligations of the regular school day and year including time and effort to reach students and families. However, according to (Keller, 2007), the high demands of time outside of the regular school day would increase the workload exponentially and cause burnout for teachers. Numerous claims of burnout add to the growing number of transiency of teachers in urban schools which would negatively impact student achievement and the relationships established with families.

The small schools initiative also has gaps in the curricula that compromises the quality of instruction by restricting teacher's autonomy to be creative and differentiate the instruction to meet the individual needs of students. The curricula does not push students beyond the basic levels of rigor and does not meet the higher levels necessary to prepare students for advanced classes in the future. There are many bright points to the small school initiative however, school leaders will have to carefully decide what aspects of the initiative that would be beneficial for their school (Keller, 2007). Once that decision is made, trust in the leadership is crucial to teachers selling the change to students and parents as a step in the right direction and in the best interest for students. 


\section{Building Trust in Leadership}

Building trust for leaders in urban schools is essential for teachers not only to buy into the direction of leadership, but to sell the initiatives and direction to external stakeholders. Trust in the leadership has been directly related to high productivity and reaching intended outcomes for organizations (Gillespie, 2004). In reference to urban school principals, the need for staff to believe in and implement the initiatives that will increase student achievement is pivotal to urban schools reaching their goals.

A transformational leader moves members of the organization from isolation to collaboration, and from compliance to commitment. This movement is only possible when leaders create opportunities and conditions conducive to high levels of collegial trust, the encouragement from leadership to take risks, engage in reflective practices, and openness to new concepts and ideas (Liethwood \& Jantzi, 1990)

In addition, Fullan adds that leadership is the driving force to large scaled performance (Fullan, 2003). However, the development of relational trust is a very difficult process. This process must be carefully led in a way that allows the change process to be implemented in stages. These stages will allow opportunities and time for faculty to build the trust necessary to have courageous conversations in difficult situations, to take risks in decision making and to feel supported when staff members are out of their comfort zones (Fullan, 2003).

Trust between leadership and faculty is a key component to effective communication. However, it is important to emphasize that trust does not come to leadership automatically. Trust is cultivated on an ongoing basis through continuous displays of respect for the ideas of others, engaging faculty in meaningful discussion, and assuring that all opinions are shared and valued (Fullan, 2003). Holding high expectations communicates trust in the competence of faculty and is an understood behavioral norm of effective leadership. As Fullan tells us, leaders must be 
purposeful with facilitating opportunities to building trust to sustain a committed culture. Maturing competence levels of teams will have the chance to emerge during professional learning opportunities.

\section{Professional Learning}

Statistics cited from the U.S. Department of Education, (1996) showed a correlation between low academic performance, socioeconomic status and the growing gap between Blacks, Hispanics and their white counterparts (Sigelman and Welch, 1991). Moreover, the projected increase in population for both racial groups only heightens most researchers' concerns and implies the need for change within the smaller structures of urban school districts at the school level.

The origins of professional learning communities began with an ethnography that showed the most significant difference between high and low performing schools was collaboration (Williams, 2013). The transformational framework encourages a shared vision that is created through collaborative efforts and collegiality. This is a paradigm shift from the traditional ways of organizational hierarchy. In the traditional setting, individuals generally worked in isolation or in silos in classrooms, grade levels and communication usually flowed from top down. The transformational framework operates from the perspective of all voices being heard and a more democratic approach that solicits feedback from all members of the organization (Jahan, 1999). Time can definitely be an issue in implementing this approach however, the transformational framework values incremental change, long-term sustainability rather than sporadic growth that erratically fluctuates randomly over time (Jahan, 1999).

Traditionally, educators received a cookie cutter or a one-size-fits-all professional development experience. The topic would cover the latest district initiative or strategy that is 
introduced as an expectation for all educators to use in classrooms (Willams, 2013). In most cases, professional developments are not differentiated or tailored to individual needs due to time, space, and finances. Teachers either leave invigorated and eager to implement strategies learned into the next lesson plan or they leave as if they have seen a movie for the third time.

Typically, the professional development experience is held for several days in the summer, usually a week or two before school and once a month for an hour for the rest of the year. The topics are not the same therefore the support is not ongoing. One-shot presentations are not conducive to gradual change (Willams, 2013). Professional learning opportunities should be ongoing which allots time for discussion or collegial conversations to occur. Presentations should be relevant to the needs of teachers and in alignment of the school's accountability plan to move forward. Next, teachers should receive descriptive feedback in a timely manner (Hoord, 2009).

Currently, urban school districts across the country are replacing these hit and miss professional development experiences with organized PLCs that are embedded within the school schedule to allow teachers to collaborate on a regular basis (Castle, Arends, \& Rockwood, 2008). Collaboration was found to be a significant factor in improving student achievement in low performing urban schools. Collaboration is pivotal in shifting the focus from teaching to learning outcomes (Castle, Arends, \& Rockwood, 2008). PLCs allow teachers to engage in discourse around effective instructional approaches, sharing of best practices, and problem solving to guide instructional next steps. Collaboration also allows teachers the opportunities to learn from each other by comparing and contrasting strategies, planning together and analyzing data to inform next steps moving forward. This is an ongoing process that will allow teachers to make adjustments in the curricula as needed (Williams, 2013).

Poverty poses many challenges for American schools around the nation. Because urban centers 
have large pockets of poor schools serving children in these centers find unique educational challenges and scarce opportunities. Chapter two begins with the literature focusing on the challenges that principals face in urban schools, the complexities of building a culture of trust in urban schools and ending by transitioning into the ongoing process of implementing professional learning communities in urban, school settings. 


\section{CHAPTER 3}

\section{METHODOLOGY}

\section{Introduction and Overview}

This chapter includes the procedures and methods used to examine leaders and faculty of secondary urban schools that provide a culture of commitment to high levels of collegiality, expectations, and professional learning. The opening section of this chapter addresses the purpose and rationale for the research design and methodology. Additional sections of this chapter include the purpose of the study, research questions, research sample of the study, an overview of information needed, the research design, the data collection methods, data analysis, synthesis, ethical considerations, issues of trustworthiness, limitations of the study and the chapter summary.

This study is considered a case study for several reasons. A case study has been defined as an approach to investigating a phenomena's real-life context (Merriam, 2001). Merriam (2001), defines a case study as an analysis of a single instance. In this research study, the phenomena would be a culture of commitment to high levels of collegiality, high expectations, and professional learning. However, this case study becomes a bounded case study because the phenomena is not clearly evident (Meriam, 2001). Moreover, the phenomena is bounded by urban secondary schools located in the Midwest in which teachers and leaders will be interviewed. This case study also explores transformational leaders that motivate staff to hold high standards for their students and themselves in challenging conditions too often found in urban communities.

\section{Purpose Statement}

The purpose of this study is to examine leaders and faculty of secondary urban schools that provide a culture of commitment to high levels of collegiality, expectations, and professional 
learning. Specifically, this research will illustrate how leaders in urban, secondary schools raise the commitment levels of teachers; show evidence of a school environment that has adapted to the challenges of poverty in urban areas and lastly, the impact of professional learning communities on the overall school culture.

A number of methodologies were examined, however, a qualitative design was chosen as the approach to inquiry as a method to view the characteristics and cultural norms of a progressive urban school culture through the lens of transformational leadership. The product of this research will include a detailed description of the problem and a signal for a call to action (Creswell, 2007).

The specific form of qualitative research that will be used is a collective case study. A collective case study is a type of case study in which the researcher selects several programs to better illustrate the problem from different perspectives. Often the researchers design the case study to use the same procedures for each program of study (Creswell, 2007). Data is collected from multiple sources including interviews, observations, documents, reports, and etcetera.

Below are the three essential research questions that will guide this investigation.

\section{Research Questions}

The research questions guiding this study are:

1. How do leaders of urban secondary schools with sustained culture of high academic performance develop collegial trust?

2. How do school leaders of urban secondary schools demonstrate academic expectations for themselves and their students?

3. How do school leaders of urban secondary schools promote a professional learning culture? 


\section{Research Sample}

\section{Site Selection}

In this section of the qualitative study, purposeful sampling was used to inform an understanding of the research problem. Events, settings, and artifacts are the strategic, main aspects of purposeful sampling according to Marshall \& Rossman (2006). In this case study, maximum-variation sampling was chosen to enable the researcher to identify secondary schools with sustained cultures of high academic performance and located in urban areas as a means to study schools. Teachers and leaders that demonstrate high academic expectations for themselves and their students were also chosen to study. Maximum-variation sampling also permits the researcher to indicate the uniqueness of each situation and the commonalities of each site or situation (Mertens, 2005). Maximum-variation sampling is an effective approach in qualitative research because it tolerates differences between sites or participants to add a variation of perspectives and experiences which will inform the study from a holistic viewpoint (Creswell, 2007). Commonalities are discovered and are then used as important data points.

The Multifactor Leadership Questionnaire (Appendix 1) was used to evaluate the leadership styles of principals. The tool allowed individuals to measure their perceptions of their leadership behaviors by utilizing a self-leadership form (Avolio \& Bass, 2004). Participants were asked to respond to 45 items which were measured by a five point behavioral scale. The questionnaire should not exceed 15 minutes (Avolio \& Bass, 2004). Principals that showed characteristics of the transformational leadership style from urban districts were selected as participants for the study to exemplify how this leadership style was ideal for sustaining a culture of high academic expectations, sustained academic performance and a professional learning culture for all. Transformational leaders seek out the motivational engines that power each individual in the organization. This motivation is paired with the overarching goals of the 
organization so that outcomes are beneficial for both member and the organization (Burns, 1978). This mutual stimulation transforms the member into a change agent that will satisfy both self and organizational goals. Information gained from this approach may give insight related to the aforementioned research questions.

Teacher leaders were selected by implementing a maximum variation of sampling technique, identifying a group of teachers that have expertise in diversity to create a heterogeneous sampling group. The criteria for selecting teacher leaders included: three years or more experience in urban and secondary education, experience working in professional learning communities, and experience with using data to drive instructional decision making. Participants verified certification, teaching experience, and specific work experiences as they applied to the purpose of this study prior to focus group.

Confidentiality of participants is a high priority for the researcher. Confidentiality is a challenge for qualitative researchers because of the rich information that is being shared by participants (Kaiser, 2009). Although the rich data results in a more in depth understanding in researching a phenomena, there are also unintended consequences. One unintended consequence of qualitative research, including case studies and focus groups, is deductive disclosure (Kaiser, 2009). Deductive disclosure occurs when traits of participants or groups such as age, gender, number of years served, and other data assessable to the public breaches the confidentiality of the research and identifies the participants (Tolich, 2004). Casual identifiers may seem to have little or no significance however, they can have a negative impact on the livelihood of the participants, the credibility of the organization and the confidence in researchers (Kaiser, 2009).

As a proactive measure to protect participant confidentiality, the researchers of this study reviewed with the participants the audience that the research findings will be shared to allow the participants a voice in the process of disseminating the information. Next, confidentiality was 
openly discussed with participants before, during and after data collection was completed. The researcher also elicited feedback from participants regarding sensitive information that should be handled with extreme care. At the conclusion of the research, a post interview confidentiality form was shared as a final layer of support to protect participant's confidentiality. The aforementioned approach is the alternative approach, introduced by Karen Kaiser (Kaiser, 2009).

\section{Overview of Information Needed}

Focus groups' effectiveness can be attributed to an increase of opportunities for researchers to seek a deeper understanding of the "why" behind rich data points shared by participants that may give further insight of the underlying root cause that cannot be fully captured through using traditional interviewing methods (Vaughn, Schumm, Sinagub, 1996). Data from focus groups often expands the researcher's knowledge base and allows the information to be more readily available, similar to surveys or polls, however focus groups provide more in depth research findings than statistical data points (Vaughn, Schumm, Sinagub, 1996).

Focus groups can be used as standalone instruments or in conjunction with other instruments such as surveys or artifacts (Vaughn, Schumm, Sinagub, 1996). Focus groups are effectively utilized in qualitative research because the interactive aspect of the small group, interviews' mode of operation offers opportunities for purposeful sampling to elicit multiple points of view to emerge and inform the origins of the thought process associated with the reported outcomes (Gulliksen, Hjardemaal, 2016). Focus groups put high value on both the moderator and the participants. They give unique opportunities for the moderator to use leverage as a probing tool for deeper explanation of abstract and intrinsic ideas of the participant as a research tool to provide clarity or extend the cognitive process in deriving individual truths (Silverman, 2016). 
For the purpose of this research, focus groups were grouped with teachers from improved secondary urban schools which are under corrective action status by the state department of education and principals from similar schools, which utilize transformational leadership methods in their practice. Both groups of educators were then be asked questions by the moderator. The moderator's primary job was to facilitate idea sharing and individual perspectives of the aforementioned research questions as well as utilizing probing questioning techniques during the group sessions as the conversations evolved. Findings from focus groups of teachers and principals were triangulated with artifact data such as meeting agendas, professional learning community data reports, meeting notes, and any other pertinent documents relative to answering the research questions.

\section{Research Design Overview}

\section{Study Participants}

For the purpose of this research, three focus groups were comprised of five to eight teachers that work in secondary urban schools that have been classified as schools under corrective action and are showing significant academic gains since the corrective action determination (Gibbs, 1997). Each school leader was interviewed separately lasting no more than two hours. The school leader met the criteria explained in the research sample section. The school selected met the criteria of a school under corrective action and evidenced significant academic gains since the corrective action determination. Each group was also asked to share any documentation or artifacts that captured the work done as part of the processes and procedures established by their respective organizations (as shown in Table 1 triangulation chart). This information was also used as a data point for the purpose of this research.

\section{Format of Focus Group Sessions}

Each session was limited to no more than two hours for the discussion of all questions 
(Gibbs, 1997). Group norms were established at the beginning of the session in which the facilitator maintained order and insured that the group remained on task. All participants were encouraged to actively participate and to keep confidentiality at the forefront of importance. The facilitator then gave an overview of the problem of practice and the guiding research questions. The discussion was recorded by an assistant moderator, who served the purpose of capturing participants' answers to questions, explanations, points of clarity, anonymously (see Table 2). The location was a centrally located venue, exhibiting the space and atmosphere conducive to the comfort levels of all participants.

The atmosphere of the venue was conducive to the purpose of gathering information in an uninterrupted manner. Participant confidentiality was explained at the onset and conclusion of the session. If at any time a participant chose to withdraw, the decision would not be questioned but respected. The findings of the data was shared with all participants at the conclusion of the research and a permission to participate form was given to all prospective participants before each session began. An engagement research technique that was determined prior to scheduling the focus groups was implemented during the sessions as well as other probing techniques identified by the researcher to ensure that all voices were heard and captured for the purpose of the research. 
Table 1.

Triangulation flow-chart
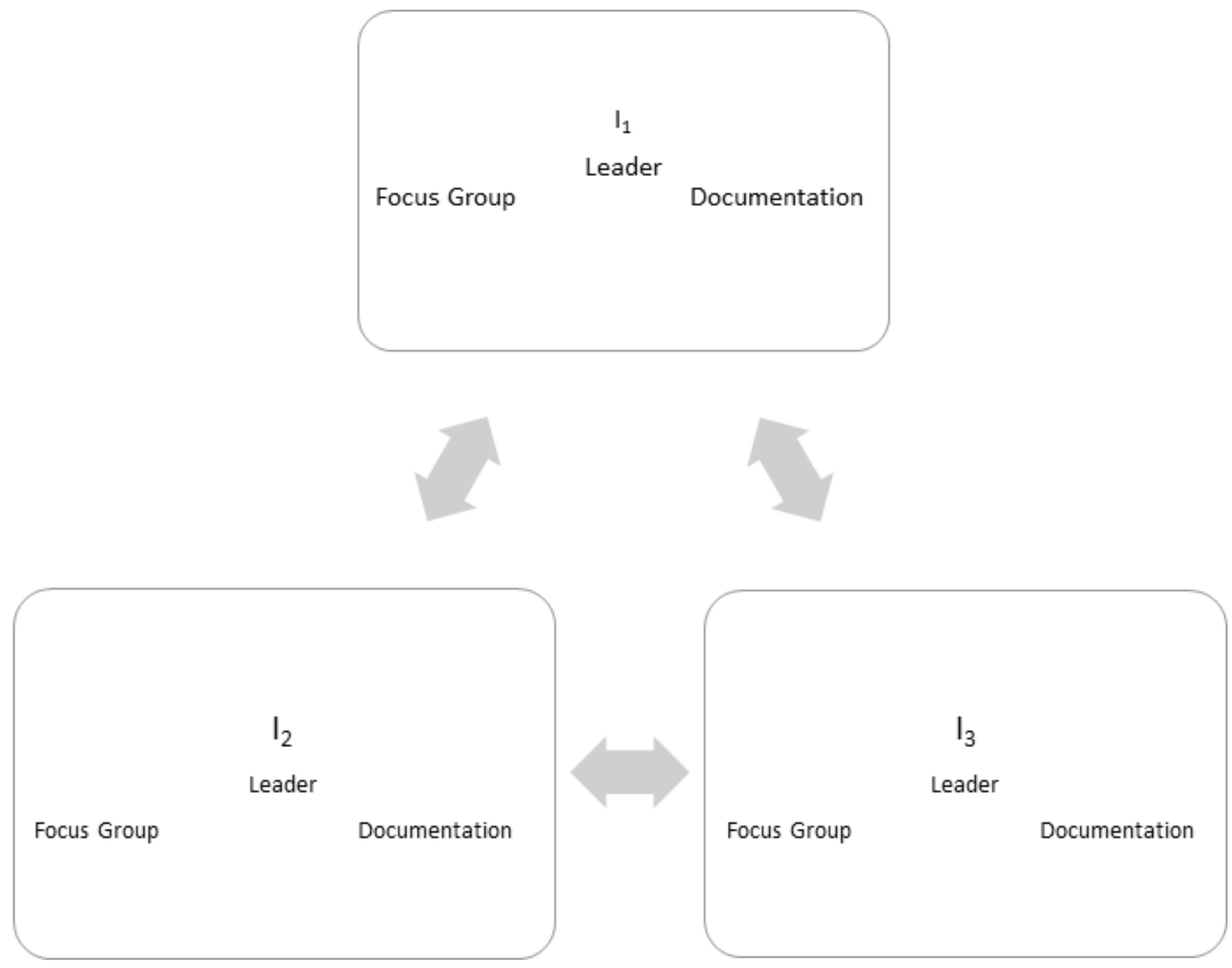
Table 2

Data summary chart

\begin{tabular}{|c|c|c|c|c|c|c|}
\hline Participant & Theme 1-1 & Theme 1-2 & Theme 1-3 & Theme 1-4 & Theme 1-5 & Theme 1-6 \\
\hline no. 1 & & & & & & \\
\hline no. 2 & & & & & & \\
\hline no. 3 & & & & & & \\
\hline no. 4 & & & & & & \\
\hline no. 5 & & & & & & \\
\hline no. 6 & & & & & & \\
\hline
\end{tabular}




\section{Data-Collection Methods}

A traditional single-category design method was implemented by using three focus groups with a targeted participants of educators that primarily service highly impoverished families in urbanized areas (Krueger and Casey, 2000). The single-category design method was used until theoretical saturation was met. Saturation is a term used to define when the data are becoming repetitive and the themes are not bringing forth new information (Krueger and Casey, 2000).

The interview questions were carefully planned so that they align to the purpose of the research. The sequence of the interview questions was an intentional process. Most traditional methods place quick, factual questions at the beginning of the interview to elicit participation from every participant in an attempt to ease the tension of the group and serve as a warm up activity.

Data collection begins immediately after the first question is asked. For the purpose of this research, the moderator was charged with asking the questions and probing for more indepth answers when deemed necessary. The moderator was also tasked with transition questions if participants are echoing the same talking points. In addition, the moderator will take notes and track the participation from each participant. This includes managing experts, dominant talkers, shy participants and ramblers (Krueger and Casey, 2002).

The second research team member focused primarily on the session recordings, field notes, key points, post-meeting debriefings with the research team, transcribing answers from the audio equipment and gathering documentation from participants. The data analysis is done concurrently with data collection. As the first focus group concluded, the data analysis process began simultaneously as the data collection of the second focus group was prepared. Analyzing 
the data in chunks while progressing through the group sessions improves the data collection process. This allows the moderator time to transcribe tapes from previous sessions and inform question effectiveness and the quality of information for future sessions. (Krueger and Casey, 2002).

The data from the first group is then compared to the second focus group. This process was repeated with the third focus group. After each focus group session was completed, interview questions were reviewed and a reflection of whether or not the effectiveness of interview questions was meeting the needs of the research study. The researcher also determined if research questions, format and techniques used were yielding insightful information from participants. Interview questions were systematically and sequentially arranged and continuously reviewed by the research team to insure that the data accurately depicted the information shared by participants.

\section{Data Analysis}

The tape based-abridged transcript method was utilized because it is a time effective approach that only requires the researcher to listen and record relevant and pertinent excerpts of the discussions (Krueger and Casey, 2014). This will simplify and shorten the arduous process of transcribing hours of irrelevant conversations by only focusing on the meaningful portions. However, this process requires the expertise of a research team member that has extensive knowledge of the research design and purpose of the study (Krueger and Casey, 2014).

In addition, the long-table approach was utilized as a simple way of analyzing transcripts and identifying themes as they emerged. This method required the researcher to secure a space to spread out excerpts of transcripts in an organized manner that allowed quotes to be arranged to begin the coding process. Transcripts were then be labeled according to focus group designation, teachers, and principals. The data was synthesized and disaggregated into categories, which 
helped identify emerging themes.

Comments were then cut from transcripts and placed in the appropriate categories. Comments were also categorized as useful to the research or not. Comments that were not deemed useful were put in a storage box marked for later viewing as a precaution to being prematurely discarded. At this time, a summary of each group's comments were written to interpret what was said thematically. Comparing and contrasting commenced between each group's comments. The researcher then determined a method of assigning weight and value to comments according to frequency, specificity, emotion, and extensiveness. Next, these steps eventually led the researcher to an overall interpretation and or a recommendation (Krueger and Casey, 2014).

\section{Ethical Considerations}

Ethical considerations for focus groups generally mirror other forms of social research (Gibbs, 1997). Researchers have to approach participants in a transparent manner. This calls for the moderator to be forthcoming with all participants of the expectations of the research purpose, audiences that the information will be shared with, timelines, recommendations and future uses of the research. The researchers are outside the participating organization and have no power or influence over the participants.

A unique ethical consideration for focus groups is confidentiality. Confidentiality is important because at any given time there is more than one person present when confidential information is being shared. Researchers should encourage participants to keep information shared during interview sessions confidential. Researchers should also communicate the participant's choice to discontinue their participation in the focus group at any time (Krueger, 2014).The aforementioned precautions were in place to protect the integrity of the research and the privacy of its participants. These precautions also aided in ensuring the credibility and 
trustworthiness of the research.

\section{Limitations of the Study}

There are many advantages to conducting focus groups such as the inclusivity of all learners including those with disabilities. Another advantage is the researcher's ability to get more in depth data from participants. The researcher has the unique ability in focus groups to follow data from the theoretical to practical aspects. This leads the researcher to a holistic understanding of the findings (Gibbs, 1997). In contrast, there are some disadvantages and limitations to conducting focus groups. Such disadvantages include response time between participants will vary and limit the amount of questions covered in a session. This limiting factor puts an emphasis on the moderator's ability to manage the pace and flow of the group. Moreover, depending on the expertise and experience of the moderator, the possibility of bias is greater if dominant participants' views are captured more often than other participants (Gulliksen \& Hjardemaal, 2016). Moderators must also capture the voices of shy and timid participants.

Confidentiality is a high risk limitation because of the rich data shared poses the threat of deductive disclosure and interactive makeup of focus groups (Krueger, 2014). The researchers must put processes in place at the onset and conclusion of the research to insure that confidentiality is a high priority. Another limitation of the research is a small sample with only a single school bounded. This poses a potential threat to validity but informs future research needed. Although small sample makes it hard for transferability of the data from one setting to the other it may inform future research designs with information to expound upon.

\section{Chapter 3 Summary}

In this research study, the phenomena investigated is a school culture of commitment to high levels of collegiality, high expectations, and professional learning. Specifically, this 
research illustrates how leaders in urban, secondary schools raise the commitment levels of teachers; show evidence of a school environment that has adapted to the challenges of poverty in urban areas and, lastly; the impact of professional learning communities on the overall school culture.

For the purpose of this research, focus groups were grouped with teachers from improved secondary urban schools which are under corrective action status by the state department of education and principals from similar schools, which utilize transformational leadership methods in their practice. Teachers were placed in focus groups and principals were interviewed separately.

The tape based-abridged transcript method was utilized as well as the long-table approach as a simple way of analyzing transcripts and identifying themes as they emerged. Confidentiality is a high risk limitation because of the rich data shared poses the threat of deductive disclosure and interactive makeup of focus groups (Krueger, 2014). Therefore, confidentiality was openly discussed with participants before, during and after data collection was completed. Feedback was elicited from participants in regards to sensitive information and a dissemination plan were shared as proactive measures to insure a high level of confidentiality. A post interview confidentiality form was utilized at the conclusion of the research. 


\section{CHAPTER 4}

\section{RESEARCH, DATA COLLECTION AND FINDINGS}

\section{Overview}

Chapter IV recaps the purpose of this qualitative study, the research questions, and the methodology used. Chapter IV presents a breakdown of the qualitative data collected from focus group interviews, three principal interviews and artifact reviews. The data describes the sustained cultures of high academic performance, located in urban areas as a means to study schools and the transformational leadership styles of each secondary principal. A summary of the key themes that emerged from the interviews and the major findings were provided.

\section{Research Purpose}

The purpose of this qualitative study is to examine leaders and faculty members of secondary urban schools that provide a culture of commitment to high levels of collegiality, high expectations, and professional learning. This research illustrates how leaders in urban, secondary schools transform the cultures of schools and raise the commitment levels of teachers while sustaining academic gains for students. This research searched for evidence of a school environment conducive to the real world challenges of urban education in the twenty first century. Lastly, this research investigated the promotion of professional learning and it becoming a viable part of the culture. Below are the three essential research questions that guided this investigation.

\section{Research Questions}

The research questions guiding this study are:

1. How do leaders of urban secondary schools with sustained culture of high academic performance develop collegial trust?

2. How do school leaders of urban secondary schools demonstrate academic expectations 
for themselves and their students?

3. How do school leaders of urban secondary schools promote a professional learning culture?

\section{Research Methods and Data Collection Procedures}

A qualitative design, purposeful sampling, was used to inform an understanding of the research problem. Events, settings, and artifacts are the strategic, main aspects of purposeful sampling according to (Marshall \& Rossman, 2006). In this case study, maximum-variation sampling was chosen to enable the researcher to identify secondary schools with sustained cultures of high academic performance. Teachers and leaders that demonstrate high academic expectations for them and their students were chosen for study.

Maximum-variation sampling also permits the researcher to indicate the uniqueness of each situation and the commonalities of each site or situation (Mertens, 2005). Maximumvariation sampling was the approach chosen because it tolerates differences between sites or participants to add a variation of perspectives and experiences, which will inform the study from a holistic viewpoint (Creswell, 2007). Commonalities are discovered and are then used as themes captured from interviews. Therefore, focus groups were chosen as a means to seek a deeper understanding of the "why" behind rich data points shared by participants that gave further insight of the underlying root causes of success that could not otherwise be fully captured through using traditional interviewing methods (Vaughn, Schumm, Sinagub, 1996). Data from focus groups often expands the researcher's knowledge base and provided more in depth research findings than statistical data points (Vaughn, Schumm, Sinagub, 1996). The focus groups allowed the researcher to gather rich quotations from participants and added deeper meaning to the perspectives of the conversations of each group. This acquired knowledge allowed the researcher to more accurately interpret findings from the qualitative data and the 
significance of the practices, thought processes and experiences shared in the focus group sessions.

The Multifactor Leadership Questionnaire (Appendix 1) was used to evaluate the leadership styles of principals. The tool also allowed individuals to measure their perceptions of their leadership behaviors by utilizing a self-leadership form (Avolio \& Bass, 2004). Participants were asked to respond to questions in which were measured by a five point behavioral scale. The questionnaire did not exceed 20 minutes. Principals that showed characteristics of the transformational leadership style and worked in urban districts informed the researcher of how and why this leadership style may be ideal for sustaining a culture of high academic expectations, sustained academic performance and a professional learning culture.

\section{Population}

For the purpose of this qualitative research, three focus groups were comprised of teachers that work in secondary urban schools. These schools are classified as schools under corrective action and showing significant academic gains since the corrective action determination (Gibbs, 1997). Focus group interviews lasted no longer than two hours. Each school leader was interviewed separately lasting no more than one hour. The school leaders met the criteria explained in the research sample section. The schools selected also were classified as schools under corrective action and are showing significant academic gains since the corrective action determination. Each group was asked to share artifacts that captured the work done to evidence the processes and procedures established by their respective organization. These artifacts are also to be used as data points to enhance perspectives and bring deeper meaning to experiences captured in interviews.

\section{Sample}

For the purpose of this qualitative study, the sample was comprised of three secondary 
principals who had completed the Multifactor Leadership Questionnaire and were interviewed by the lead researcher. This maximum variation sampling provided the researcher with profound and rich conversations, which respected the differences between participants to add a variation of perspectives, and experiences, which informed the study from a holistic viewpoint (Creswell, 2007). A total of 15 certified secondary teachers that currently work in urban educational settings participated in focus groups.

For the purpose of this qualitative research, focus groups were chosen because of the unique ability to be used in conjunction with artifact data (Vaughn, Schumm, Sinagub, 1996). Focus groups are effectively utilized in qualitative research because the interactive aspect of the small group interview's modality offers opportunities for purposeful sampling to elicit multiple points of view to emerge based on the moderators ability to use leverage as a probing tool for deeper explanation of abstract ideas of the participant as a research tool to extend the cognitive process in deriving individual truths (Silverman, Gulliksen, Hjardemaal, 2016). These focus groups were comprised of teachers from improved secondary urban schools under corrective action status by the state department of education and principals from the schools who utilize transformational leadership methods in their practice. Both groups of educators were then asked questions by the moderator. The moderator's primary job is to facilitate idea sharing and capture individual perspectives of the aforementioned research questions as well as utilizing probing questioning techniques during the group sessions as the conversations evolve.

The focus group participants teaching experience ranged from three to thirty years, averaging eleven years of experience in education at a secondary school located in an urban setting. Eleven of the participants of the focus groups are in the position of teacher, ranging from three to twenty seven years of teaching experience and averaging twelve years of teaching experience at a secondary school located in an urban setting. Ten of the eleven 
participants in the focus groups were female and one was male.

Four participants were instructional coaches located in one of the three schools in the study with experience in education ranging from seven to thirty years and averaging eighteen years of experience in education. All instructional coaches were female. The three principals in the study administrative experience ranged from eight to fourteen years and averaging eleven years of administrative experience at a secondary school located in an urban setting. Of the three principals, two were male and one was female.

Table 3

Data for Administrative Interviews

\begin{tabular}{llll}
\hline Participant & Gender & $\begin{array}{l}\text { Secondary } \\
\text { Experience }\end{array}$ & Number of Years \\
Sharron & Female & Principal, (Middle) & 11 \\
Fred & Male & Principal, (High) & 14 \\
Tamarcus & Male & Principal, (High) & 8 \\
\hline
\end{tabular}

\section{Table 4}

Data for Focus Interview Groups

\begin{tabular}{llll}
\hline Participant & Gender & Secondary Experience & Number of Years \\
\hline Everlee & Female & Instructional Coach & 30 \\
Ethel & Female & Teacher/Team Leader & 27 \\
Lilian & Female & Teacher & 15 \\
Christine & Female & Teacher & 5 \\
Naevell & Female & Instructional Guide & 20 \\
Shirley & Female & Special Education & 4 \\
Sheila & Female & Teacher & 4 \\
Sharon & Female & Teacher & 11 \\
\hline
\end{tabular}




\begin{tabular}{llll}
\hline Yvonne & Female & Instructional Coach & 7 \\
Donise & Female & Coach/Department Head & 13 \\
Anaya & Female & Teacher & 5 \\
Neal & Male & Sped. Teacher & 12 \\
Elaine & Female & Team Leader & 6 \\
Mertis & Female & Teacher & 5 \\
Sharronda & Female & Teacher & 3 \\
\hline
\end{tabular}

\section{Recruitment Process}

The study was introduced via email to teachers working at schools that met the criteria for the study. The criteria for selecting teacher leaders included: three years or more experience in urban and secondary education, experience working in professional learning communities, and experience with using data to drive instructional decision making. Participants verified certification, teaching experience, and specific work experiences via responses from the recruitment email. An invitation to participate was sent to all potential participants via email, by the research investigator explaining the purpose, confidentiality and minimal risk. Participants were approached and consented by the research investigator in a public gathering place.

\section{Focus Group Interview Process}

The researcher encouraged participants to keep information shared during interview sessions confidential. The researcher also communicated the participants' choice to discontinue their participation in the focus group at any time (Krueger, 2014).

Confidentiality of participants is a high priority. Participants were not asked to introduce their names and pseudonyms were 
used when referring to the group in the research findings to protect any identities. As a proactive measure to protect participant confidentiality, the researcher of this study reviewed with the participants that the research findings would be shared to allow the participants a voice in the process of disseminating the information.

Next, confidentiality was openly discussed with participants before, during and after data collection was completed. At the conclusion of the research, a post interview confidentiality form was shared as a final layer of support to protect participant's confidentiality. The aforementioned approach is the alternative approach introduced by Karen Kaiser (Kaiser, 2009). The research interviews took place at a public venue. All questions asked in each focus group were the same. The focus group participants were asked nineteen open ended questions regarding their practical experiences revolving around the central topics of professional learning communities, trust building, and collaboration. In addition, focus group participants were asked questions regarding their perceptions of the culture of high academic success for students. Follow-up questions preceded as an effort to encourage reflection in order to gain a deeper understanding of ideas shared by focus group participants in a manner that did not influence the participant's responses. This approach captured data directly from participants' quotes (Husbands 2017).

\section{Principal Interview Process}

Principals were asked to respond to forty five items measured by a five point behavioral scale (1 low and 5 high). The multifactor leadership questionnaire did not exceed twenty minutes (Avolio \& Bass, 2004). Principals that showed characteristics of the transformational leadership style from urban districts were selected as participants for the study to exemplify how this leadership style was ideal for sustaining a culture of high academic expectations, sustained 
academic performance and a professional learning culture for all.

In the interview, the principals were asked nineteen open ended questions regarding their practical experiences revolving around the central topics of professional learning communities, trust building, and collaboration. Moreover, principals were asked questions regarding their perceptions of the culture of high academic success for students. Probing questions preceded as an effort to encourage reflection in order to gain a deeper understanding of ideas shared by focus group participants in a manner that did not influence the participant's responses. This approach captured data directly from participant's quotes (Husbands 2017).

\section{Multifactor Leadership Questionnaire}

The Multifactor Leadership Questionnaire was used to evaluate the leadership styles of principals (see Appendix A). The tool allowed individuals to self-measure their perceptions of their leadership behaviors by utilizing a self-leadership form (Avolio \& Bass, 2004). Three multifactor leadership questionnaires were analyzed and gave a deeper insight on each principals' leadership style. The Multifactor Leadership Questionnaire measures an individual's leadership by using seven factors that lead to transformational leadership. Each factor is measured by totaling specified items on the questionnaire (Dumdum 2013). Participants scoring in the range of 9-12 are rated as high. Participants scoring in the range of 5-8 are rated as moderate. Participants scoring in the range of 0-4 are rated as low. Table 5 shows the range in which each participant scored in all seven transformational factors. The names in the chart are pseudonyms. 


\section{Table 5}

MLQ Transformational Factor Chart

\begin{tabular}{|l|l|l|l|l|l|l|l|}
\hline $\begin{array}{l}\text { Participant } \\
\text { (Principals) }\end{array}$ & $\begin{array}{l}\text { Idealized } \\
\text { Influence }\end{array}$ & $\begin{array}{l}\text { Inspirational } \\
\text { Motivation }\end{array}$ & $\begin{array}{l}\text { Intellectual } \\
\text { Stimulation }\end{array}$ & $\begin{array}{l}\text { Individual } \\
\text { Consideration }\end{array}$ & $\begin{array}{l}\text { Contingent } \\
\text { Rewards }\end{array}$ & $\begin{array}{l}\text { Management } \\
\text { by Exception }\end{array}$ & $\begin{array}{l}\text { Laissez- } \\
\text { Faire } \\
\text { Leadership }\end{array}$ \\
\hline Sharron & 12 High & 10 High & 10 High & 11 High & 11 High & 10 High & 4 Low \\
\hline Fred & $10 \mathrm{High}$ & 11 High & 12 High & 11 High & 9 High & 9 High & 6 Mod. \\
\hline Tamarcus & 11 High & 10 High & 11 High & 12 High & 7 Mod. & 10 High & 1 Low \\
\hline
\end{tabular}

The first factor is idealized influence. Idealized influence is an indicator of trust building with subordinates. Terms associated with this factor are faith, trust, respect, dedication and role model. The second factor is inspirational motivation. Inspirational motivation is an indicator of being a visionary. Terms associated with this factor are vision, symbols, images, focus, and empowerment. The third factor is intellectual stimulation. Intellectual stimulation is an indicator of encouraging others to become reflective learners. Terms associated with this factor are reflection, innovation, goal setting, differentiation, and out of the box thinking. The fourth factor is individualized consideration. Individualized consideration is an indicator of embracing all members of the organization. Terms associated with this factor are inclusivity, empowerment and honoring all voices (Dumdum 2013). The fifth factor is contingent reward. Contingent reward is an indicator of setting clear expectations, transactional and rewarding accomplishments. Terms associated with this factor are expectations, reward, recognition and accomplishments. The sixth factor is management by exception. Management by exception is an indicator of meeting the minimal standard and maintaining the status quo. Terms associated with this factor are meet the standard status quo, sustain, and maintain. The seventh factor of the multifactor leadership questionnaire is laissez-faire. Laissez-faire is an indicator of a hands off approach, minimal 
expectations. Terms associated with this factor hands off, laid back and content (Dumdum 2013).

\section{Artifact Collection}

Various documents were requested from the teachers and principals. The requested materials originated from either the teachers or principals or from data warehouses such as internal hard drives, school websites, emails, excel spreadsheets or email blasts communicated from the schools. The criterion was that the documents submitted had to be created by the participants. All documents received electronically were converted to a portable document format and saved on the researcher's secured laptop computer. Documents that were received in paper form were digitally scanned and saved on the researcher's secured laptop computer.

\section{Table 6}

Artifact Data

\begin{tabular}{lc}
\hline \multicolumn{1}{c}{ Artifacts } & Quantity \\
\hline Data Trackers/Organizers & 10 \\
Professional Development Agendas & 4 \\
PLC agenda/action plans & 6 \\
Instructional Leadership Team Meeting Minutes & 4 \\
Collegial Learning Walk Schedules & 3 \\
Principal's Monthly email blast & 2 \\
Total & 26 \\
\end{tabular}


Ten documents were excel spreadsheets in which the academic achievement data was stored and aggregated. Four professional development agendas dated from the 2016-2017 school year were analyzed. The professional development agendas evidenced the various professional development topics staff explored throughout the school year as a means of continuous improvement. Six professional learning community agendas/ action plans were analyzed. The agendas/action plans evidenced instructional planning, best practice sharing based on data results and next steps for the direction of classroom instruction. Three collegial learning walk schedules were analyzed and evidenced a building wide practice of teachers observing each other's classrooms and discussing data collected, individual interpretations and implementation of initiatives or strategies connected to professional development topics covered.

Two email blasts created by two of the three principals interviewed demonstrated evidence of communication with internal and external stakeholders' academic achievement expectations and the status of several assessments, calendar of upcoming events and celebrations and acknowledgements of staff students, and parents. Several email blasts shared monthly status reports or interpretation of specific skill strands of benchmark data from core academic subject areas which included English, Mathematics and Science. Eblasts also showed longitudinal data points in the same areas and upcoming assessment dates for students as well as celebrating individual growth from students. Walk through data was only shared internally to show trend data to teachers, students and staff and also substantiate future professional development topics and the building wide problem of practice as a way moving forward. Wording used in eblasts did not directly encourage risk taking, language used emphasized procedures for gathering, 
interpreting and sharing data.

\section{Data Analysis}

After each focus group interview and individual principal interview, the lead researcher uploaded the audio recording to a transcription application. A secured account with a private password was created so that only the researcher had access to the account. Within 24 hours of each interview, the audio files were downloaded from the transcription application and converted to a word document on the researchers' secured laptop computer. The interview transcriptions were then reviewed and edited for any errors due to noise recorded or words lost in transcription.

Once transcriptions were in a readable format, they were emailed to participants as an opportunity to receive feedback regarding the accuracy of collecting the thoughts and ideas shared in each focus group and interview. After feedback was received from all research participants, the transcriptions were finalized and saved on the researchers' secured laptop computer.

The long-table approach was utilized as a simple way of analyzing transcripts and identifying themes as they emerged. This method required the researcher to secure a space to spread out excerpts of transcripts in an organized manner that will allow quotes to be arranged to begin the coding process. Transcripts were then labeled according to focus group designations of: The Fab 4, The Go Getters, and The Sunshine Band. The data was then synthesized and disaggregated into categories, which helped the process of identifying emerging themes.

Comments were then cut from transcripts and placed in the appropriate categories. Comments were also categorized as useful to the research or not. Comments that were not deemed useful were put in a storage box marked for later viewing. A summary of each group's comments was written to interpret what was said thematically. Comparing and contrasting then commenced 
between each groups' comments. The researcher then determined a method of assigning weight and value to comments according to frequency, specificity, emotion, and extensiveness. The five themes that emerged from focus groups were, autonomy, data driven/growth, collaboration, professional development, and trust.

Table 7

Focus Group Thematic Frequency chart

\begin{tabular}{|c|c|c|c|c|c|}
\hline $\begin{array}{c}\text { Focus } \\
\text { Group }\end{array}$ & $\begin{array}{l}\text { Theme } \\
\text { Autonomy } \\
\text { Orange }\end{array}$ & $\begin{array}{c}\text { Theme } \\
\text { Data } \\
\text { Driven/Growth } \\
\text { Yellow }\end{array}$ & $\begin{array}{l}\text { Theme } \\
\text { Collaboration }\end{array}$ & $\begin{array}{c}\text { Theme } \\
\text { Professional } \\
\text { Development }\end{array}$ & $\begin{array}{l}\text { Theme } \\
\text { Trust }\end{array}$ \\
\hline Fab 4 (1) & 9 & 17 & 21 & 25 & 18 \\
\hline $\begin{array}{c}\text { Go Getters } \\
\text { (2) }\end{array}$ & 15 & 41 & 30 & 46 & 28 \\
\hline $\begin{array}{c}\text { Sunshine } \\
\text { Band } \\
(3)\end{array}$ & 7 & 16 & 14 & 20 & 13 \\
\hline $\begin{array}{c}\text { Total } \\
\text { (All) }\end{array}$ & $\mathbf{3 1}$ & $\mathbf{7 4}$ & $\mathbf{6 5}$ & $\mathbf{9 1}$ & $\mathbf{5 9}$ \\
\hline
\end{tabular}

Table 7 reveals the frequency of emerging themes from each collective focus group interview. The first column represented the theme autonomy, which had a total of 31 references made during the focus groups. Autonomy can easily be linked to risk taking as described by 
Boleman and Deal in the literature review (chapter two). The transformational leadership style encourages risk taking as an accepted part of the organizational culture. Transformational leaders are innovative thinkers that plan with the end in mind, predict unintended consequences of decision making and empower employees to gain relevant experiences (Bolman \& Deal, 2010). The second column represented the theme data driven/growth, which had a total of 74 references made during the focus groups. Williams shares that collaboration allows teachers the opportunities to learn from each other by comparing and contrasting strategies, planning together and analyzing data to inform next steps moving forward. This is an ongoing process that will allow teachers to make adjustments in the curricula as needed (Williams, 2013).

The third column represented the theme collaboration, which had a total of 65 references made during the focus groups. Researchers cited in the literature review (chapter two) indicated that PLCs are communities of professionals learning collaboratively in an ongoing, inquiry based, analytical approach for continuous improvement. Information is shared through best practices, data driven practices and a respect for differences within community (Lindsey, Jungwirth, Pahl, 2009).

The fourth column represented the theme professional development, which had a total of 91 references made during the focus groups. This was by far the largest amount of references made under any of the themes during focus groups. Williams shared that one-shot presentations are not conducive to gradual change (Willams, 2013). The topics are not the same, therefore, the support is not ongoing. Professional learning opportunities should be ongoing, which allots time for discussion or collegial conversations to occur. Presentations should be relevant to the needs of teachers and in alignment of the school's accountability plan to move forward.

The fifth column represented the theme trust, which had a total of 59 references made 
during the focus groups. Building supportive relationships with children, teachers, school, home and the community at large will make the difference in establishing a culture conducive to teaching and learning (Comer, 1988). Relationships will also minimize barriers between school and external stakeholders. Trust between leadership and faculty is a key component to effective communication. However, it is important to emphasize that trust does not come to leadership automatically. Trust is cultivated on an ongoing basis through continuous displays of respect for the ideas of others, engaging faculty in meaningful discussion, and assuring that all opinions are shared and valued (Fullan, 2003).

The primary form of data collection was the open-ended interview. However, the researcher prepared an interview guide to strategically preserve a basic structure to this informal, conversational approach in interviewing. The interview guide eventually was a resource with organizing and analyzing the data (Bowen 2005). There were several patterns, themes and categories that eventually emerged from the data. This approach is also known as the inductive method. The inductive methos was a logical fit for the data collection method for the purpose of the research (Bowen 2005).

For the qualitative phase of the research, data collected from the focus group interviews revealed five themes that were consistent from each session. The themes included autonomy, data driven/growth, collaboration, professional development, and trust.

Autonomy. Participants gave rich conversations around the theme of autonomy. Participants were asked: Is risk taking encouraged?

Everlee (teacher) described how risk taking was encouraged in instructional decisions:

Absolutely the map rally (academic pep rally) as an example today; we take risks you 
know and if it doesn't work we pick ourselves back up and we plan better for next year. Anything that is put into place like he the principal or the leader of the building he just allows us to jump on board like if he says we're planning a map rally who wants to be on board with that and we have our own voice with it he didn't give use any clip cut way of what he wanted we were able to do our own thing it we think it would have worked absolutely sometimes you just never know.

Ethel (see Ethel quote) also shared that risk taking was highly encouraged in the planning and facilitating process of professional development meetings:

I think teachers take risk in facilitating PD's. I would say teachers in our building are encouraged to take risk when you are working with curriculums and people becoming comfortable in their content area, and our principal pushing and saying that you have the autonomy, you are the expert in the classroom. So, sometimes, all teachers are given the autonomy to make some of the curriculum decisions that may not always happen in a place where risks are a part of any evaluation.

Lilian noted that her principal has given teachers at her school the autonomy to schedule classes based on the individual needs of students:

We've also been able to group our students; if you look at our student groupings they changed mid-year because we noticed that some students have made gains and not be with us anymore and some students may need leave; we look at our groupings throughout the year you will see that those are flexible as well. So if you asking for evidence that we can show you and you will see that; also she has allowed us to group our students in a way that one teacher may have low, one teacher may have mid, one teacher may have high. So we don't have all these different levels of students that we work with and we 
can focus in on what that particular group of students need. Our structure for what the reading specialist may look like, may look different and it came from us and it wasn't top down although we have to stay within the parameters of the district, we make sure to do that. It's not like we are just randomly scheduling students.

In total contrast of Everlee and Ethel, Christine, shared that there were several cases of autonomy but it is managed and granted with parameters:

I will say it [risk taking] is encouraged but to an extent because I feel like at certain times we will be encouraged to do new things here techniques that work in the classroom but then when it gets closer to maybe like say testing or when something happens or some kind of switch then leadership may say if it's not broke don't try to fix it, keep doing what you been doing. So I would say it's to a certain extent; they want you to take risk but at the same time not really.

Naevell, a teacher from the same school and focus group added that risk taking is encouraged but better described as calculated risks:

I'm going to say it is encouraged cautiously because if you are in tested areas and they're proven methods that another teacher did and it worked before regardless of the fact of the students who came before they want it to work for the kids that follow; so it is cautiously encouraged if you're not teaching in a tested course you have more freedom to explore and try new things there's no test that follows if it fails you can go back and reteach and spend more time on the skill verses in a class that has an exam at the end.

Data driven/growth. The next theme, data driven/growth, showed a significant increase in references from all participants in each focus group. When asked about the primary academic 
expectation of the leadership, data and growth were consistently mentioned across the board. Shirley shared:

Anytime that they give an incentive for something you know it is important. So there's incentives for attendance, there is incentives for growth on a practice test, there is incentives for making proficient or advanced so anytime someone gives you an incentive for something you know that's where the priority is.

Shiela added her experience with the leadership continuously checking and watching the data and basing next steps on outcomes or data trends:

They're checking on it and following through. So for example so with the attendance thing the attendance is outside of the main office, also outside of the social worker office the kids are encouraged to know their own personal data there is a follow-through on two levels the student has to know their own information and then the leadership has to keep track or the I guess like you said follow through keeping it update new information for each kid in the building that's testing.

Sharon gave a different outlook on academic expectations. She began by acknowledging the negative effects of poverty on education, and spoke to relationship building and meeting holistic needs as the first steps towards academic achievement:

She [the principal] understands some of the baggage [effects of poverty] that the student bring and that we don't just address the academic needs. Her primary expectation is that students move, make movement it's not always to me what I've gotten from her, it's not always about the necessarily having to be on grade level, it's about them showing progress, showing growth throughout the year and that's what it should be I mean if you can have high expectations but some things only God can take care of in a year; I mean, 
so, her thing is showing progress and because of that our students do show progress because it's not "well you have to be here by the end of the year"; it is we want you to grow as a person and as a student academically and behaviorally she doesn't just focus on academics it's behavior as well; she focus on the whole child.

The researcher then asked a probing question to solicit a more in depth response. The researcher asked the participants to explain how academic expectations was communicated and what academic expectations looked like. Yvonne immediately explained how academic expectations were communicated:

We talk about it [academic data] all the time. Professional development, it's on the announcements, during staff meetings, it's during any type of meeting that we have with leadership you're going to hear about these different topics of things that are reiterated over and over. So you know that it is something that is important because it is constantly said and it is constantly demonstrated in a way that the leadership handles the discipline data trends in the school as well as the parents that they have to deal with.

Collaboration. The next emerging theme was collaboration, the researcher found evidence of collaborative practices in the responses of another focus group in a different interview question. The group was asked about the method of academic expectations being communicated. Donise (teacher) gave a list of several opportunities facilitated by the school leadership:

Through collaboration, meetings, emails, and walkthroughs. Grade level meetings, team walkthroughs, district walkthroughs, rallies, and instructional coaches. The building is the professional learning community; they [teachers] meet in collaborative teams to examine data and to develop shared instructional strategies. Meetings occur consistently once a week and I feel like we go over the four corollary questions just basically talking when 
we bring student data what students know and what will we do if they haven't achieved. So basically, having the conversations and sometimes even from my team we meet once a week but sometimes those conversations happen in the hallway while we're in transition and taking our kids to specials [electives]. I feel like I'm always checking in with my team; like what worked well today so we constantly have conversations each and every day, even though we meet once a week as a team. We really set the focus for the week and we are okay being flexible sometimes we can't stick to the schedule so we make an adjustment if students aren't ready to move forward. We have the conversations and we as a team, adjust the curriculum.

Anaya (teacher) expounded upon the response by adding how collaborative practices have become a viable part of what teachers do every day. She explained how collaboration not only occurs in formal settings but in informal settings as well. Anaya added:

I can speak for me I would say yes because each day like I said before we have conversations every day in passing so it's not just sitting down for our weekly meeting we are constantly talking about what's in place for each day; so I can constantly want to check in with my team to make sure they have what they need and we talk throughout the day; about the math lesson what went well do you feel like we need to back up and do this over same thing with science and social studies there are a lot of new initiatives this school year I feel teachers need a lot of support because there's at least two new programs and next school year I think reading will be new teachers need a lot of support so I think there should be a lot of check-ins because we're not familiar with it so I guess that's for everybody.

Neal (teacher) shared that his opportunities for collaboration came in the form of best practice 
sharing. Neal shared an example of an instructional strategy that could be implemented across the curricula:

I think in staff meetings we are able to share what works best for us teachers are able to present. I know that in the past teachers have presented lessons based off of things that have worked well. A Japanese lesson study shared was a kindergarten piece, but it was something that can be utilized at other grade levels in the district to help teachers to see best practice, to reflect on best practice and to make decisions about how they thought they would implement it differently. Best practices are also shared during our walkthroughs. I have observed teachers teaching lessons and received a book from her on a lesson. I was like oh this is amazing and she was like here this is an extra one you can use in your class. Ms. J. also gave me writing materials; another teacher gave me stuff for writing; and again it was through that particular walkthrough experience that I began to incorporate strategies observed in my class.

Elaine (teacher) added the need for more job embedded opportunities for collaborative time with other grade levels. While she was appreciative of the time allocated for same grade level meetings, she expressed concerns for lack of time to meet with vertical teams:

I feel the need to express for more opportunities to have with vertical collaborative teams in our building. Our schedule is created in a way that all third grade teachers have the same plan. While this is helpful, our team is also in need of more time to be protected in our building calendar for dialogue between the grade levels.

Professional Development (PD): The next theme is professional development, which is the theme most heavily referenced by all participants. There were several aspects of PD on which participants focused. The first was relevance. Mertis began with the prescribed PD and then 
transitioned into individualized PD:

Every month we get a half-day of PD, and if you are on one of the district's initiatives there are PD once a month for that. It depends on where you are and what you want to commit to because it is voluntary. However, in our building teachers are so committed that they participate in all the committees. Another issue with professional development I think it is important to note that they allow the teachers to lead the PD In the past, the district used to pay a lot of presenters to come in, and I felt like it was wasted money. You have valuable educators who are knowledgeable and they are allowing the teachers to grow; so you are growing your own teachers to become leaders, therefore; I think that's really good; when you promote the teachers in the building, they know what's needed so it's not a lot of wasted time. Before we were going to all these Pds. and we were frustrated; it was like "why are we here; what is going on; is this going to benefit my students?" So you making me sit through something that's not valuable and it's not worth my time.

The researcher asked another probing question to gain a deeper understanding. The researcher asked participants about opportunities to give feedback for professional development relevant to their needs. Once again, Mertis (teacher), led the discussion by sharing her experiences with online surveys:

Survey Monkey was an online survey in which we responded to our needs, our strengths, what we wanted to work on, our weaknesses, and there's a response where you can typein anything else. The district would put it all together for all to see. Based off of that, workshops were created along with the summer institute. This was the PD we have had in the district and I have been here for 17 years. The teacher lead PD is more meaningful 
because when you put a teacher in charge you get so much more. Surveys were issued prior to the workshop and it asked you which ones you wanted to attend. Lastly, you had the opportunity to give feedback if it was something not beneficial. Allowing teacher work time to apply whatever they learned during the day is vital. All the information with time to plan or practice is very useful for teachers. I think gradual release is really important to have embedded in our professional developments.

Trust. The last theme that emerged from the research study was trust. The data was slightly different from each group. The differences were dependent upon style, approach and relationships. Sharronda (teacher) gave insightful feedback when asked by the researcher about leadership being approachable:

She's [the principal] very open; she's very welcoming and she listens. She's very approachable to me, she listens, she welcomes ideas, she trusts everyone to be professionals and she's realistic about the children we service and that's first and foremost, understanding the culture of this building. I feel like I'm harder on myself than she is on us; she's like you have to understand where the kids are coming; from some of the needs and she's very understanding about that and she trusts us. She really trusts us to make the decisions and it is no topic or subject that she will not discuss with you. I mean she doesn't have a problem discussing any subject, you can be mad, crying, she will still talk to you; there's nothing that will deter her from tough conversations. She doesn't take it personal, it's just business this is what we do. We [reading teachers] got to the point where we can just run our own meeting; she is going to listen to what we have to say; to our D and C and she will have A and B; she trust our skill set and what we bring to the table and based on what we've done in past she knows we are going to make it happen; she doesn't have to hold our hand. 
Anaya (teacher) shared thoughts on how her principal is a straightforward, communicator that is logic based and a proven instructional leader:

I think his [principal] reputation kind of speaks for itself, you see the growth, you see the change, you see what he's been able to do as far as transforming the school date, numbers, accreditation, and all of that. You see it, so you trust in that, well I guess he is right so whatever he's doing is working. He's pushing the school to go, its working so let me just do my part and shut up.

Table 8 represents the thematic frequency chart from the principal interviews. There is a correlation between the emphasis that both teachers and principals placed on professional development rather than data. I made the assumption that the data process identified the need for improvement in a given area, however, the opportunity for professional development allowed teachers and principals to learn new strategies to implement and take action which would seem more fulfilling or beneficial than the data process of crunching numbers and disaggregating data points. 
Table 8

Thematic Frequency Principal Interview chart

\begin{tabular}{|c|c|c|c|c|c|}
\hline Focus Group & & $\begin{array}{l}\text { Theme } \\
\text { Autonomy } \\
\text { Data } \\
\text { Driven/Growth }\end{array}$ & $\begin{array}{c}\text { Theme } \\
\text { Collaboration }\end{array}$ & $\begin{array}{c}\text { Theme } \\
\text { Professional } \\
\text { Development }\end{array}$ & $\begin{array}{c}\text { Theme } \\
\text { Trust }\end{array}$ \\
\hline $\begin{array}{c}\text { Sharron } \\
\text { (Middle) }\end{array}$ & 3 & 13 & 8 & 14 & 4 \\
\hline $\begin{array}{c}\text { Fred } \\
\text { (H.S.) }\end{array}$ & 7 & 15 & 12 & 21 & 11 \\
\hline $\begin{array}{c}\text { Sunshine } \\
\text { (H.S.) }\end{array}$ & 2 & 8 & 2 & 7 & 9 \\
\hline Total & 12 & 36 & 22 & 42 & 24 \\
\hline
\end{tabular}

The first column represented the theme autonomy, which had a total of 12 references made during the principal interviews. The second column represented the theme data driven/growth, which had a total of 36 references made during the principal interviews. The third column represented the theme collaboration, which had a total of 22 references made during the principal interviews. This was the largest amount of references made under any of the themes during the principal interviews. The fourth column represented the theme professional development, which had a total of 42 references made during the principal 
interviews. The fifth column represented the theme trust, which had a total of 24 references made during the principal interviews. The primary form of data collection for the principal interviews was also the open-ended interview. The interview guide was consistent with the one made for teachers in design as a means to strategically preserve a basic structure to this informal conversational approach in interviewing. The interview guide was a resource with organizing and analyzing the data (Bowen 2005). The themes included autonomy, data driven/growth, collaboration, professional development, and trust.

Autonomy. Beginning with autonomy, participants gave rich conversations around this theme. Principals were asked: Do you encourage risk taking?

Sharron explained that risk taking a healthy part of trial and error when searching for answers:

Risk taking is a healthy part of trial and error in research and education. Teachers need to feel that they have been empowered and have the autonomy to experiment with ideas, strategies, and innovative approaches to teaching and learning. Especially in today's classrooms. Educators are now asked to not only step out of the box but to blow up the box!

Fred stated that risk taking occurs once trust is built. The trust building process begins with the leadership:

It is the responsibility for the leadership to facilitate opportunities for staff learn through exploration of new, cutting edge, research based, teaching strategies that engage students and push their learning to higher levels of rigor and thinking. This will include experimentation and failure. However, short termed failure may lead to long term success. Tamarcus gave his vision of risk taking as a non-evaluative, trial and error 
period that allows teachers time to meet the academic needs of students:

Risk taking should be a part of the coaching process to building capacity and strengthening skill sets. My leadership team is good at modeling, collegial walks, and reflective practices that will allow teachers to analyze their own practices and compare them to best practices shared. This process broadens their perspectives as well as adds tools in their box of strategies to increase learning.

Data Driven/Growth Tamarcus explains how everything that teachers do should be data driven, examine trends, and continue the process:

All instructional decisions should point back to the data. The data leads us to potential solutions and the data should also lead us away from ineffective strategies, initiatives, and practices. The data tells us where we are and leads us to next steps instruction.

Fred asserts that data can be a four lettered word in education because data driven decision making is not openly embraced by teachers:

Although data driven decision making is not a new concept in education, it is viewed as a new phenomenon used by principals to torture teachers. There is a clear discomfort with teachers using data to substantiate instructional decisions. This is another skill set that teachers are not proficient at coming out of college. This is not a trend but a way of life especially in underperforming districts.

Sharron explains that growth is incremental in most cases, therefore, the data should reflect that incremental growth when significant gains are not apparent:When analyzing data, growth should be noted and celebrated incrementally and individually. Each student is different and goals should be set in chunks that are obtainable and realistic. This will approach will serve as an 
ongoing motivator for students and staff in obtaining long-term goals.

Collaboration. Fred shares how collaboration gives us a new lens to view challenges. Often times it also gives us a new perspective or outlook on an outdated practice that we have been stuck with:

Collaboration allows teachers to play off one another with new ideas; teachers are able to grow from each other and work together to come up with common formative assessments and grade them together as they analyze the data, the results of assessments and make informed decisions based on data and their newfound collaborative understanding.

Sharron shares that collaborative opportunities for staff should be embedded within the academic calendar:

If the leadership protects collaborative time and emphasizes its' importance by creating operational procedures as well as expected outcomes or next steps from meetings then staff will begin to make this time a priority in their work day.

Tamarcus explained that collaboration, although not a new way of thinking in education, can be a paradigm shift for some:

In education often times we get settled in our individual classrooms, create a subculture different from the schools' and operate in total isolation. We become territorial of our classroom, we hoard materials and unfortunately, information including best practices. Collaboration is the crucial element to our continued professional growth. The concept is simple, when you bring more minds to the thinking tank the outcome usually yields successful results.

Professional Development. Fred explained the process that he follows to elicit feedback 
regarding professional development needs and the direction of professional development moving forward:

When I observe trends in classrooms that are related to instruction during walk-throughs, that information informs me of potential professional development opportunities for staff as a way to support them instructionally. Next, I would create a walkthrough template based on the problem of practice and share it with the building leadership team as a way to calibrate look-fors and how they will be measured during classroom walkthroughs. The leadership team would then create an action plan timeline and begin to observe classrooms. Systematically, we will cover the building and comeback together and analyze the data. We would identify trends and share the findings with the staff. This would then inform our next steps for building professional development. A professional development calendar would be created and we would begin professional development, best practice sharing, etcetera. We would then revisit classrooms as a check for improvement and allow the data to drive the continued steps for support and improvement.

Trust. Tamarcus described some of the strategies that he uses to build trust with his staff, students and stakeholders:

There are several strategies and techniques that I use to break down barriers and begin to make people comfortable communicating with me. I begin this process by facilitating opportunities for stakeholders to communicate with me and I act as an active listener. I am conscious of body language, gestures, follow up questions, documenting concerns, and giving any push back. After the feedback sessions, I analyze the data and begin to code trends and rank them according to the number of references made to each. I then 
report back to stakeholders and begin to address the problems communicated, celebrate the success and create an action plan to continue the process of open communication. Another strategy used is high visibility. I make an effort to be in well-traveled areas so that I am accessible. I also use several modes of communication to ensure that all communication preferences are used. Descriptive feedback is another strategy that I am progressing on. I have found that everyone enjoys feedback. This something that I challenge myself to do more each year as a leader.

Fred shared that trust building is a process that requires patience, listening, and an investment of time:

Trust building requires the patience to listen to staff concerns before and after work or during busy moments in your schedule. This includes on the parking lot, on the way to work or home from work. Responding to long emails of concern, attending staff resolution meetings, parent meetings, community meetings and being transparent with information. Trust building is a process that requires an investment of time and effort before stakeholders begin to feel comfortable enough to trust the leadership and the direction of the organization.

\section{Chapter 4 Summary}

Chapter 4 covered a recap of the purpose of this qualitative study, the research questions, and the methodology used. Chapter IV also presented a breakdown of the qualitative data collected by means of three focus group interviews, three principal interviews and artifact reviews to describe the sustained cultures of high academic performance, located in urban areas as a means to study schools and the transformational leadership styles of each secondary principal. 
The focus group participants teaching experience ranged from 3 to 30 years, averaging 11 years of experience in education at a secondary school located in an urban setting. Ten of the eleven participants in the focus groups were female and 1 was male. The three principals in the study administrative experience ranged from 8 to 14 years and averaging 11 years of administrative experience at a secondary school located in an urban setting. Of the three principals, 2 were male and 1 was female.

The Multifactor Leadership Questionnaire was used to evaluate the leadership styles of principals (see Appendix A). The tool allowed individuals to self-measure their perceptions of their leadership behaviors by utilizing a self-leadership form (Avolio \& Bass, 2004). The tape based-abridged transcript method was utilized because it is a time effective approach that only requires the researcher to listen and record relevant and pertinent excerpts of the discussions (Krueger and Casey, 2014). There were a total of 26 documents analyzed by the researcher. Findings, limitations, and next steps for the research in the future will be shared in detail, in chapter 5 . 


\section{Chapter 5}

\section{Research Findings, Conclusions and Recommendations}

Chapter V provides a review of the purpose of this qualitative study, three guiding research questions, research methodology, target population, and research sample. Chapter V then submits a summary of the significant findings of the data collected. Lastly, conclusions formulated by the lead researcher based on all findings inform implications for further action and future research recommendations. Chapter V concludes with reflections and conclusions from the researcher.

\section{Research \\ Purpose}

The purpose of this qualitative study is to examine leaders and faculty of secondary urban schools that provide a culture of commitment to high levels of collegiality, high expectations, and professional learning. This research illustrates how leaders in urban, secondary schools transform the cultures of schools and raise the commitment levels of teachers while sustaining academic gains for students. This research searched for evidence of a school environment conducive to the real world challenges of the twenty first century urban education. Lastly, this research also investigated the promotion of professional learning and its becoming a viable part of the culture. Below are the three essential research questions that guided this investigation.

\section{Research Questions}

The research questions guiding this study are:

1. How do leaders of urban secondary schools with sustained culture of high academic performance develop collegial trust? 
2. How do school leaders of urban secondary schools demonstrate academic expectations for themselves and their students?

3. How do school leaders of urban secondary schools promote a professional learning culture?

\section{Research Methods and Data Collection Procedures}

A qualitative design was used to inform an understanding of the research problem. Events, settings, and artifacts are the strategic, main aspects of purposeful sampling according to (Marshall \& Rossman, 2006). In this case study, maximum-variation sampling was chosen to enable the researcher to identify secondary schools with sustained cultures of high academic performance. Teachers and school leaders that demonstrate high academic expectations for themselves and their students were also chosen to study.

Maximum-variation sampling also permits the researcher to indicate the uniqueness of each situation and the commonalities of each site or situation (Mertens, 2005). Maximumvariation sampling was the approach chosen because it tolerates differences between sites or participants to add a variation of perspectives and experiences which will inform the study from a holistic viewpoint (Creswell, 2007). Commonalities are discovered and are then labeled as themes captured from interviews after saturation occurs.

Therefore, focus groups were chosen as a means to seek a deeper understanding of the "why" behind rich data points shared by participants that gave further insight of the underlying root causes of success that could not otherwise be fully captured through using traditional interviewing methods (Vaughn, Schumm, Sinagub, 1996). Data from focus groups often expands the researcher's knowledge base and provided more in depth research findings than statistical data points (Vaughn, Schumm, Sinagub, 1996). The focus groups allowed the researcher to gather rich quotations from participants and added deeper meaning to the 
perspectives of the conversations of each group. This acquired knowledge allowed the researcher to more accurately interpret findings from the qualitative data and the significance of the practices, thought processes and experiences shared in the focus group sessions.

The Multifactor Leadership Questionnaire (Appendix A) was used to evaluate the leadership styles of principals. The tool also allowed individuals to measure their perceptions of their leadership behaviors by utilizing a self-leadership form (Avolio \& Bass, 2004).

Participants were asked to respond to questions which were measured by a five point behavioral scale. The questionnaire did not exceed 20 minutes. Principals that showed characteristics of the transformational leadership style and worked in urban districts informed the researcher of how and why this leadership style may be ideal for sustaining a culture of high academic expectations, sustained academic performance and a professional learning culture.

\section{Population}

For the purpose of this qualitative research, three focus groups were comprised of teachers that work in secondary urban schools that have been classified as schools under corrective action and are showing significant academic gains since the corrective action determination (Gibbs, 1997). Focus group interviews lasted no longer than two hours. Each school leader was interviewed separately lasting no more than one hour. The school leaders met the criteria explained in the research sample section. Each group was also asked to share artifacts that captured the work done to evidence the processes and procedures established by their respective organization. Artifacts will also be used as data points to enhance perspectives and bring deeper meaning to experiences captured in interviews.

\section{Sample}

For the purpose of this qualitative study, the sample was comprised of three secondary 
principals in urban settings who had completed the Multifactor Leadership Questionnaire and were interviewed by the lead researcher. This maximum variation sampling provided the researcher with profound and rich conversations which respected the differences between participants to add a variation of perspectives and experiences which informed the study from a holistic viewpoint (Creswell, 2007). A total of 15 certified secondary teachers, which currently work in urban educational settings, participated in focus groups.

For the purpose of this qualitative research, focus groups were chosen because of the unique ability to be used in conjunction with artifact data (Vaughn, Schumm, Sinagub, 1996). Focus groups are effectively utilized in qualitative research because the interactive aspect of the small group interview's modality offers opportunities for purposeful sampling to elicit multiple points of view to emerge based on the moderators ability to use leverage as a probing tool for deeper explanation of abstract ideas of the participant in order to extend the cognitive process in deriving individual truths (Silverman, Gulliksen, Hjardemaal, 2016).

For the purpose of this research, focus groups were grouped with teachers from improved secondary urban schools which are under corrective action status by the state department of education and the principals from the schools which utilize transformational leadership methods in their practice. Both groups of educators were then asked questions by the moderator. The moderator's primary job is to facilitate idea sharing and individual perspectives of the aforementioned research questions as well as utilizing probing question techniques during the group sessions as the conversations evolves.

The focus group participants teaching experience ranged from 3 to 30 years averaging 11 years of experience in education at a secondary school located in an urban setting. Eleven of the participants of the focus groups are in the position of teacher ranging from 3 to 27 and averaging 12 years of teaching experience at a secondary school located in an urban setting. 
Ten of the eleven participants in the focus groups were female and 1 was male.

Four participants were instructional coaches located in one of the three schools in the study with experience in education ranging from 7 to 30 years and averaging 18 years of experience in education. All instructional coaches were female. The three principals in the study had administrative experience ranging from 8 to 14 years and averaging 11 years of administrative experience at a secondary school located in an urban setting. Of the three principals, 2 were male and 1 was female.

\section{Research Findings}

A case study is defined as an approach to investigating a phenomena's real-life context (Merriam, 2001). Merriam (2001), defines a case study as an analysis of a single instance. In this research study, the phenomena would be a culture of commitment to high levels of collegiality, high expectations, and professional learning. However, this case study becomes a bounded case study because the phenomena is not clearly evident (Meriam, 2001). Moreover, the phenomena is bounded by urban secondary schools located in the Midwest in which teachers and leaders will be interviewed. This case study also explores transformational leaders that motivate staff to hold high standards for their students and themselves in challenging conditions too often found in urban communities.

For the purpose of this research, focus groups were grouped with 15 teachers from improved secondary urban schools which are under corrective action status by the state department of education and 3 principals from the schools which utilize transformational leadership methods in their practice.

All groups of educators were then asked questions by the researcher. Each group was also asked to share any documentation or artifacts that captured the work done as part of the processes and procedures established by their respective schools (as shown in Table 1 
triangulation chart). The analysis of the data revealed the experiences from all 18 participants, along with artifact data which further illustrated how leaders utilize transformational strategies to motivate staff to create a culture of commitment to high levels of collegiality, high expectations, and professional learning.

\section{Finding 1: Collaboration is a Key Element to Decision Making Relative to Teaching and Learning in Urban Secondary Schools.}

The qualitative data collected from focus group interviews, artifacts, and principal interviews demonstrated how transformational leaders motivate staff to create a culture of commitment to high levels of collegiality. The conclusion is based on the finding that $100 \%$ of all focus groups shared experiences that evidenced collaboration as a key element to decision making relative to teaching and learning in urban, secondary schools. Moreover, focus groups had a total of 65 references made during the focus groups to collaboration, which made up $20 \%$ of all references to themes that emerged from focus group interviews (see Table 7 in Chapter IV).

Donise (teacher) gave a list of several opportunities facilitated by the school leadership:

Through collaboration, meetings, emails, and walkthroughs. Grade level meetings, team walkthroughs, district walkthroughs, rallies, and instructional coaches. The building is the professional learning community; they [teachers] meet in collaborative teams to examine data and to develop shared instructional strategies.

Meetings occur consistently once a week and I feel like we go over the four corollary questions just basically talking when we bring student data what students know and what will we do if they haven't achieved. So basically, having the conversations and sometimes even from my team we meet once a week but sometimes those conversations 
happen in the hallway while we're in transition and taking our kids to specials [electives]. I feel like I'm always checking in with my team; like what worked well today so we constantly have conversations each and every day, even though we meet once a week as a team. We really set the focus for the week and we are okay being flexible sometimes we can't stick to the schedule so we make an adjustment if students aren't ready to move forward. We have the conversations and we as a team, adjust the curriculum.

Anaya (teacher) expounded upon the response by adding hoe collaborative practices have become a viable part of what teachers do every day. She explained how collaboration not only occurs in formal settings but in informal settings as well. Anaya added:

I can speak for me I would say yes because each day like I said before we have conversations every day in passing so it's not just sitting down for our weekly meeting we are constantly talking about what's in place for each day; so I can constantly want to check in with my team to make sure they have what they need and we talk throughout the day; about the math lesson what went well do you feel like we need to back up and do this over same thing with science and social studies there are a lot of new initiatives this school year I feel teachers need a lot of support because there's at least two new programs and next school year I think reading will be new teachers need a lot of support so I think there should be a lot of check-ins because we're not familiar with it so I guess that's for everybody.

Finding 1 is also based on the finding 19 of the 26 artifacts analyzed by the researcher showed evidence that collaboration is a key element to decision making relative to teaching and learning in urban secondary schools. This totaled to $73 \%$ of all artifacts analyzed from research 
participants (see Table 6 in Chapter IV).

Next, the aforementioned finding is based on $100 \%$ of all principal interviews' shared experiences that collaboration is a key element to decision making relative to teaching and learning in urban secondary schools. In addition, principal interviews had, during the interviews, a total of 22 of references made to collaboration, which made up $16 \%$ of all references to themes that emerged from principal interviews (see Table 8 in Chapter IV).

Lastly, literature also supports the first finding as collaboration was found to be a significant factor in improving student achievement in low performing urban schools. Limited opportunities for collaboration and collegial work can cause a feeling of being disconnected or isolation for teachers (Payzant, 2011). When time is not embedded within the academic schedule, teachers can become isolated from other teaching perspectives, strategies and best practices that could eventually result in frustration, cause stagnation and end in burnout. Teachers new to the profession are especially at risk when not supported and nurtured. Insufficient support in the first few years of teaching usually results in turnover over 30 percent of the time in urban schools (Payzant, 2011).

Collaboration is pivotal in shifting the focus from teaching to learning outcomes (Castle, Arends, \& Rockwood, 2008). Collaboration allows teachers the opportunities to learn from each other by comparing and contrasting strategies, planning together and analyzing data to inform next steps moving forward. This is an ongoing process that will allow teachers to make adjustments in the curricula as needed (Williams, 2013).

\author{
Finding 2: Data Driven Decisions/ Growth and Professional Development are Key \\ Elements to Decision Making Relative to Teaching and Learning in Urban Secondary \\ Schools.
}


The qualitative data collected from focus group interviews, artifacts, and principal interviews demonstrated how transformational leaders motivate staff to create a culture of commitment to high levels of professional learning. Finding 2 is based on the conclusion that $100 \%$ of all focus groups shared experiences that data driven decisions, student growth and professional development are key elements to decision making relative to teaching and learning in urban secondary schools. Moreover, focus groups had a total of 165 references made during the focus groups to data driven decisions, student growth and professional development which makes up $52 \%$ of all references to themes that emerged from focus group interviews (see Table 7 in Chapter IV).

When asked about the primary academic expectation of the leadership, data and growth was consistently mentioned across the board. Shirley shared:

Anytime that they give an incentive for something you know it is important. So there's incentives for attendance, there is incentives for growth on a practice test, there is incentives for making proficient or advanced so anytime someone gives you an incentive for something you know that's where the priority is.

Shiela added her experience with the leadership continuously checking and watching the data and basing next steps on outcomes or data trends:

They're checking on it and following through. So for example so with the attendance thing the attendance is outside of the main office, also outside of the social worker office the kids are encouraged to know their own personal data there is a follow-through on two levels the student has to know their own information and then the leadership has to keep track or the I guess like you said follow through keeping it update new information for each kid in the building that's testing. 
The conclusion is also based on the finding from 19 of the 26 artifacts analyzed by the researcher showing evidence that data driven decisions, student growth and professional development, are key elements to decision making relative to teaching and learning in urban secondary schools. This totaled to $73 \%$ of all artifacts analyzed from research participants (see Table 6 in Chapter IV).

Next, the aforementioned conclusion is based on the finding that $100 \%$ of all principal interviews shared experiences that data driven decisions, student growth and professional development, are key elements to decision making relative to teaching and learning in urban secondary schools. In addition, principal interviews had a total of 78 references made during the interviews regarding data driven decisions, student growth and professional development, which made up $57 \%$ of all references to themes that emerged from principal interviews (see Table 8 in Chapter IV).

Fred explained the process that he follows to elicit feedback regarding professional development needs and the direction of professional development moving forward:

Systematically, we will cover the building and comeback together and analyze the data. We would identify trends and share the findings with the staff. This would then inform our next steps for building professional development. A professional development calendar would be created and we would begin professional development, be st practice sharing, etcetera. We would then revisit classrooms as a check for improvement and allow the data to drive the continued steps for support and improvement.

Lastly, literature also supports the conclusion that data driven decisions, student growth 
and professional development were found to be significant factors in improving student achievement in low performing urban schools. Traditionally, educators receive a cookie cutter or a one-size-fits-all professional development experience. The topic would cover the latest district initiative or strategy that is introduced as an expectation for all educators to use in classrooms (Willams, 2013). In most cases, professional learning opportunities are not differentiated or tailored to individual needs due to time, space, and finances.

One-shot presentations are not conducive to gradual change (Willams, 2013). Professional learning opportunities should be ongoing which allots time for discussion or collegial conversations to occur. Presentations should be relevant to the needs of teachers and in alignment with the school's accountability plan to move forward. Currently, urban school districts across the country are replacing these hit and miss professional development experiences with organized PLCs that are embedded within the school schedule to allow teachers to collaborate on a regular basis (Castle, Arends, \& Rockwood, 2008). Collaboration was found to be a significant factor in improving student achievement in low performing urban schools.

\section{Finding 3: Trust and Autonomy are Key Elements to Decision Making Relative to Teaching and Learning in Urban Secondary Schools.}

The qualitative data collected from focus group interviews, artifacts, and principal interviews demonstrated how transformational leaders motivate staff to create a culture of commitment to high levels of professional learning. The conclusion is based on the finding that $100 \%$ of all focus groups shared experiences that trust and autonomy are key elements to decision making relative to teaching and learning in urban secondary schools. Moreover, focus groups had a total of 90 references made during the focus groups relevant to trust and autonomy, which makes up $28 \%$ of all references to themes that emerged from focus group 
interviews (see Table 7 in Chapter IV).

The conclusion is also based on the finding of 22 of the 26 artifacts analyzed by the researcher showing evidence that trust and autonomy are key elements to decision making relative to teaching and learning in urban secondary schools. This totaled to $85 \%$ of all artifacts analyzed from research participants (see Table 6 in Chapter IV).

Next, the aforementioned conclusion is based on the finding that $100 \%$ of all principal interviews shared experiences that trust and autonomy as key elements to decision making relative to teaching and learning in urban secondary schools. In addition, principal interviews had a total of 36 references made during the interviews regarding trust and autonomy which made up $26 \%$ of all references to themes that emerged from principal interviews (see Table 8 in Chapter IV).

Lastly, literature also supports the finding that trust and autonomy were found to be significant factors in improving student achievement in low performing urban schools. Trust in the leadership has been directly related to high productivity and reaching intended outcomes for organizations (Gillespie, 2004). A transformational leader moves members of the organization from isolation to collaboration, and from compliance to commitment. This movement is only possible when leaders create opportunities and conditions conducive to high levels of collegial trust, the encouragement from leadership to take risks, engage in reflective practices, and openness to new concepts and ideas (Liethwood \& Jantzi, 1990).

In addition, Fullan adds that leadership is the driving force to large scaled performance (Fullan, 2003). However, the development of relational trust is a very difficult process. Trust is cultivated on an ongoing basis through continuous displays of respect for the ideas of others, engaging faculty in meaningful discussion, and assuring that all opinions are shared and valued (Fullan, 2003). Holding high expectations communicates trust in the competence of faculty and 
is an understood behavioral norm of effective leadership. As Fullan tells us, leaders must be purposeful with facilitating opportunities to building trust to sustain a committed culture. Maturing competence levels of teams will have the chance to emerge during professional learning opportunities.

Building supportive relationships with children, teachers, school, home and the community at large will make the difference in establishing a culture conducive to teaching and learning (Comer, 1988). Trustworthy relationships will continuously assist in minimizing barriers between school and external stakeholders.

\section{Unexpected Findings}

A few unexpected findings were discovered from the data analysis process during this research study:

1. Teachers in the Sunshine Band focus group shared several times that "it's a lot of separate teams throughout the building." One teacher shared that her time was spent "mostly in my nook; I'm mostly up here around the same people and three to five teachers." "I'm only up here so outside the four adults, I see up here I don't really interact with any other adults. I think that it's cliques because it's like a stepbrother you like them but you don't. We are very isolated." This was a surprising finding because several researchers have found that best practice shows that isolation is detrimental to cultures of sustained academic success. For example, Leithwood

and Jantzi tell us that inner city secondary schools are particularly vulnerable to isolated departmental level structures that often separate teachers from the rest of the school and as a consequence, yields a by-product of a poorly managed infrastructure. This structure may encourage collaboration within the department but that collaboration does not extend to the general mission of that school (Andy Hargreaves as cited in Leithwood \& Jantzi, 1990). With 
the absence of a school-wide culture for excellence, the "superglue" critical to joining people to meaningful school outcomes will not occur (Bolman \& Deal, 2010). Fullan explains that as a repercussion of isolation, teachers are driven by cynicism and frustration (Fullan, 2008). What was also unexpected was that this school continued to show substantial academic gains for several years. The Sunshine Band represented a high school which may account for the feeling of isolation through the departmentalization of curricular areas.

2. The second unexpected finding was again from the Sunshine Band focus group. During the interview, several teachers shared how professional leadership committees were not a vital part of the collaborative opportunities at their school. Teachers shared:

“We don't have daily PLCs. I haven't been to a PLC since probably like November. That's the process; what it's supposed to be but it's different by department. So, her department is different than mine so they probably actually do that. It depends on whether you are teaching a tested course or not."

Moreover, tables 7 and 8, in chapter four, both show that the Sunshine Band and their principal made significantly less references to collaboration that the other focus group participants or principals participating in the interviews. Although there were some references made regarding collaboration, professional learning communities were not the main mode of collaboration for this particular school. This was a shocking and an unexpected finding. This finding is in contrast from Williams' claim that PLCs allow teachers to engage in discourse around effective instructional approaches, sharing of best practices, and problem solving to guide instructional next steps. William's continues his claim by adding that collaboration also allows teachers the opportunities to learn from each other by comparing and contrasting strategies, planning together and analyzing data to inform next steps moving forward. This is an ongoing process that will allow teachers to make adjustments in the curricula as needed 
(Williams, 2013).

\section{Conclusions}

Based on the findings of this study, the following conclusions were made based on focus group interviews, artifacts, the multifactor leadership questionnaire and principal interviews.

\section{Conclusion 1}

Secondary principals who participated in the interviews valued relationships, and empowerment which was evidenced through leadership development. The data collected from focus group interviews, artifacts, the multifactor leadership questionnaire and principal interviews demonstrated that secondary principals who participated in the interviews valued relationships and empowerment which was evidenced through leadership development.

This conclusion is based on the finding that $100 \%$ of the principals scored extremely high on factor 4 of the MLQ, individualized consideration (see Table 5). Individualized consideration indicates the degree to which you shows interest in others' well-being, assign projects individually, and pay attention to those who seem less involved in the group (Avolio 2012). In fact, one principal scored a 12 out of 12 possible and the other two principals scored 11 of 12. Moreover, focus groups referenced professional development for a combined total of 91 times, which was the highest number of references made. In addition, principals referenced professional development for a combined total of 42 times, which was also the highest number of references

made by that particular group. Individualized consideration was also evidenced in the study by Bolman and Deal in which transformational leaders are described as innovative thinkers that plan with the end in mind, predict unintended consequences of decision making and empowers 
employees to gain relevant experiences that are both aligned to their personal goals and the overarching goals of the organization (Bolman \& Deal, 2010).

\section{Conclusion 2}

Teachers are encouraged to bring innovative ideas to classrooms, take calculated risks in decision making and given autonomy within parameters given by leadership. The data collected from focus group interviews, artifacts, the multifactor leadership questionnaire and principal interviews demonstrated that teachers are encouraged to bring innovative ideas to classrooms, take calculated risks in decision making and given autonomy within parameters given by leadership.

This conclusion is based on the finding that $100 \%$ of the principals scored extremely high on factor 3 of the MLQ, intellectual stimulation (see Table 5). Intellectual stimulation is an indicator of encouraging others to become reflective learners (Avolio 2012). Terms associated with this factor are reflection, innovation, goal setting, differentiation, and out of the box thinking. In fact, one principal scored a 12 out of 12 possible and the other two principals scored 11 , and 10 of 12 possible points.

However, focus groups and principals referenced autonomy as a low priority. The data presents a slight disparity in the findings, however, further information shared by the Sunshine Band focus group suggests principals are apprehensive in giving full autonomy in decision making. The research of Liethwood and Jantzi, clearly encourages risk taking and autonomy by explaining a transformational leader as one who moves members of the organization from isolation to collaboration, and from compliance to commitment. This movement is only possible when leaders create opportunities and conditions conducive to high levels of collegial trust, the encouragement from leadership to take risks, engagement in reflective practices, and openness 
to new concepts and ideas (Liethwood \& Jantzi, 1990).

\section{Conclusion 3}

Transformational leaders are the driving force for motivating staff to create a culture of commitment to high levels of collegiality, high expectations, and professional learning. The data collected from focus group interviews, artifacts, the multifactor leadership questionnaire and principal interviews demonstrated that transformational leaders are the driving force for motivating staff to create a culture of commitment to high levels of collegiality, high expectations, and professional learning.

This conclusion is based on the finding that $100 \%$ of the principals scored relatively high on all 7 of the 8 factors of the MLQ, with the exception of Laissez- Faire Leadership (see Table 5). This conclusion is also based on the finding that themes that emerged from focus groups were autonomy, data driven, student growth, professional development, collaboration and trust, which are all terms synonymous with the description of characteristics exemplified by transformational leaders.

The artifact data included: academic data reports, professional learning community meeting notes, school improvement plans, email blasts, and board of education reports, confirmed the themes and information shared. They consistently are a part of the practices and culture in all three secondary urban schools that participants of this research study are associated with. Lastly, the research of several authors captured in the literature review further concurs with the aforementioned conclusion.

Fullan shared that leadership is the driving force to large scaled performance (Fullan, 2003). The Andersons described the transformational approach as one that creates moments of discomfort that need to be carefully managed in order to yield positive outcomes (D. Anderson \& Anderson, 2011). It will require transformational leaders to communicate a clear vision for 
the school and to sustain that vision of success on a long-term basis. Tipu adds that leader inspired motivation eventually leads members of the organization to take initiative in problem solving and to produce positive and profitable outcomes for both parties (Tipu, S.A., 2012).

\section{Implications for Action}

The data collected from focus group interviews, artifacts, the multifactor leadership questionnaire and principal interviews revealed significant findings for the development of new teacher preparation programs in secondary urban school districts, leadership development at the school district and university levels as well as trainings and or courses that emphasize the effects of generational poverty on urban education. Findings from this study produced three implications for action that are as follows:

1. The experiences shared by the fifteen teachers that participated in the focus groups indicated that the learned experiences from everyday interactions with students, parents and community members have given them a deep understanding, a new perspective, and a lens

which has proven beneficial as an urban educator. The circumstances of poverty contributes to the challenges in urban education's lack of parental involvement and student's basic health needs not met, which may lead to special educational modifications and accommodations for students of low income (Wilson 2011). External distractions are also circumstances of poverty affecting school culture and climate such as neighborhood crime, behavioral disruptions in classrooms, and a lack of financial capital. Research on urban education commonly concludes that teachers bring deficit attitudes with them due to the scarcity of cultural exposure and blame for the shortcomings of children and families that live in poverty (Battey \& Megan, 2016). On average, students spend significantly more time with teachers than with family. (Wentzel, 2009). In urban schools, teachers are charged with 
cultivating meaningful relationships with students and families as they are a crucial influence on student's social, mental and academic development. The first recommendation is for school districts and universities to collaborate in preparing aspiring teachers and administrators for experiences that will facilitate exposure to communities and families in urban settings. These experiences will provide background knowledge of the challenges of poverty and will provide a perspective beneficial to both teachers and administrators embarking upon a career as a secondary urban educator.

2. The experiences shared by the fifteen teachers and three principals that participated in the focus groups indicated that the learned experiences from opportunities created by the principal to collaborate with colleagues in the process of lesson plan development, data teams and best practice sharing will provide a much-needed support to the novice secondary teacher and administrator to implement in order to close the growing educational gap between students in urban settings and their white counterparts in affluent high performing school districts. Research concurs with this implication for action. Payzant shared that limited opportunities for collaboration and collegial work can cause a feeling of being disconnected or isolation for teachers (Payzant, 2011). When time is not embedded within the academic schedule, teachers can become isolated from other teaching perspectives, strategies and best practices that could eventually result in frustration, cause stagnation and resulting in burnout. Teachers new to the profession are especially at risk when not supported and nurtured. Insufficient support in the first few years of teaching usually results in turnover over 30 percent of the time in urban schools (Payzant, 2011). The second recommendation is for school districts and principals serving in urban settings to set aside time in the academic calendar for teachers to collaborate with each other as teams, vertically and otherwise to share best practices, participate in collegial walkthroughs and data teaming. This time would also 
allow for rich conversations with parents and guardians to share pertinent information about their children that would assist teachers in lesson preparation, behavior management and to meet the holistic needs of students. Time should also be allocated for administrators to collaborate and share best practices regarding effective practices, management versus instructional leadership, as well as, time to meet with parents and other stakeholders. 3.Another challenge for principals is trust. In the past decade, the role of the principal has drastically evolved from a managerial role to an instructional leader role (Dhuey \& Smith, 2014). Transformational leaders are innovative thinkers that plan with the end in mind, predict unintended consequences of decision making and empower employees to gain relevant experiences that are both aligned to their personal goals and the overarching goals of the organization (Bolman \& Deal, 2010). Building trust with the staff, community, and students is seemingly an insurmountable task for principals in urban schools. Trust between leadership and faculty is a key component to effective communication of concerns, goals, needs and high levels of collegiality. The third recommendation is that district level professional learning opportunities should be afforded to urban secondary principals as a layer of support to build skill sets necessary to transform the culture, academic outcomes, and build trust amongst staff in low performing, urban secondary schools.

\section{Recommendations for Future Research}

As previously stated in the study, urban education has both internal and external challenges that place inner city students at an extreme disadvantage to acquiring educational equity and access to knowledge and skills necessary to compete in a constantly evolving global society (Oakes, Franke, Quartz, Rogers, 2002). An even greater challenge is demands of highstakes testing and pressure on urban educational systems and educators to meet the holistic 
needs of their children. Consequently, teachers and administrators are tasked to close the growing educational gap between urban students of color and their white counterparts in high performing school systems (Layton, 2015). High-stakes reform creates the arduous task for principals to orchestrate a paradigm shift in thinking for all members of the organization. Based on the findings of this study, the following recommendations for further research are offered:

1. Conduct a more in-depth study with an enlarged sample group of urban educators from large urban centers across the country. The current study was bounded to schools located in the Midwest. The larger pool of educators from a larger section of the country would increase the reliability and validity of the tools used in the study.

2. The current study required principals to self-administer the MLQ (see appendix A). As of the 2017-2018 school year, the Missouri Department of Elementary and Secondary Education (DESE), made it mandatory for districts to incorporate characteristics of transformational leaders in the principal's evaluative process. This could possibly be another approach to assess the effectiveness of implementation of transformational strategies.

3. Conduct a mixed method study (qualitative and quantitative) with the additional sample group mentioned in recommendation number one. Further research could include a quantitative approach to determine specific occurrences or the effectiveness of transformational leaders with a predetermined number of transformational strategies implemented. The focus group interviews (quantitative) could be similarly charted as in this study. The quantitative data could then analyzed and checked for correlations. 


\section{Reflections and Conclusions}

Conducting this study propelled several significant findings to the forefront. It is my perception that there is a significant potential for this study to affect educational leadership decision making in underperforming urban secondary school districts. This research will contribute information to urban school leaders that are in the beginning stages of building staff capacity and creating a collaborative culture of committed educators. This study will also inform aspiring urban educators of the many challenges of secondary schools in urban settings are faced with on a daily basis such as generational poverty, a lack of financial and resources, lowered expectations, deficit attitudes, recruiting and retention challenges of teachers for principals, and inspiring overworked and underpaid staff to name a few.

Urban education has been a life-long passion of mine as I am a product of many hard working and dedicated urban educators. This study has made me more conscious of the many challenges that students in urban areas courageously face every day inside and outside of school. It also has heightened my awareness of the preparation necessary for aspiring educators needed to transform underperforming schools into high performing lighthouses of the community in the quest to close the achievement gap perpetuated by poverty, antiquated teaching practices and a disenfranchised population. 


\section{References}

Afshari, M., Bakar, K. A., Luan, W. S., \& Siraj, S. (2012). Factors Affecting the Transformational Leadership Role of Principals in Implementing ICT in Schools. Turkish Online Journal of Educational Technology - TOJET, 11(4), 164-176.

Anderson, D., \& Anderson, L. A. (2010). Beyond change management: How to achieve breakthrough results through conscious change leadership. John Wiley \& Sons.

Avolio, B. J., Zhu, W., Koh, W., \& Bhatia, P. (2004). Transformational leadership and organizational commitment: Mediating role of psychological empowerment and moderating role of structural distance. Journal of Organizational Behavior, 25(8), 951968. Retrieved from http://search.proquest.com/docview/224879573?accountid=12104

Avolio, Bruce J., and Bernard M. Bass. "Multifactor leadership questionnaire (MLQ)." Mind Garden (2004).no. 4 (Jul 2012), p. 461-480.

Anyon, J. E. A. N. (2005). A political economy of race, urban education, and educational policy. Race, identity, and representation in education, 369-378.

Bass, B. M., Avolio, B. J., \& Goodheim, L. (1987). Biography and the Assessment of Transformational Leadership at the World-Class Level. Journal of Management, 13(1), 7.

Biag, M. (2016). A Descriptive Analysis of School Connectedness: The Views of School Personnel. Urban Education, 51(1), 32-59.

Bolman, L. G., \& Deal, T. E. (Eds.). (2010). Reframing the path to school leadership: A guide for teachers and principals. Corwin Press. 
Boyd, D., Grossman, P., Ing, M., Lankford, H., Loeb, S., \& Wyckoff, J. (2011). The influence of school administrators on teacher retention decisions. American Educational Research Journal, 48(2), 303-333.

Boyd, D., Lankford, H., Loeb, S., \& Wyckoff, J. (2005). The draw of home: How teachers' preferences for proximity disadvantage urban schools. Journal of Policy Analysis and Management, 24(1), 113-132.

Bowen, G. A. (2005). Preparing a qualitative research-based dissertation: Lessons learned. The Qualitative Report, 10(2), 208-222.

Bush, T. (2009). Leadership development and school improvement: contemporary issues in leadership development. Educational review, 61(4), 375-389.

Carter, J. H., \& Keiler, L. S. (2009). Alternatively certified teachers in urban small schools: Where policy reform meets the road. The Urban Review, 41(5), 437.

Comer, J. P. (1984). Home-School Relationships as They Affect the Academic Success of Children. Education and urban society, 16(3), 323-37.

Comer, J. P. (1988). Educating poor minority children. Scientific American, 259(5), $42-48$.

Currie, G., Boyett, I., \& Suhomlinova, O. (2005). Transformational Leadership within Secondary Schools in England. A Panacea for Organizational Ills? Public Administration, 83(2), 265-296. doi:10.1111/j.0033-3298.2005.00450.x

Dhuey, E., \& Smith, J. (2014). How school principals influence student learning. 
Dorman, E. H. (2012). Teaching for Social Justice and Equity in Small Urban High Schools: Challenges and Possibilities. Online Yearbook of Urban Learning, Teaching, and Research, 1-14.

DuFour, R., \& Eaker, R. (Eds.). (2010). On common ground: The power of professional learning communities. Solution Tree Press.

Dumdum, U. R., Lowe, K. B., \& Avolio, B. J. (2013). A meta-analysis of transformational and transactional leadership correlates of effectiveness and satisfaction: An update and extension. In Transformational and Charismatic Leadership: The Road Ahead 10th Anniversary Edition (pp. 39-70). Emerald Group Publishing Limited.

Ezaki, J. Y. (2015). Secondary administrators' perceptions of the blended coaching model on their development as transformational leaders (Order No. 3685459). Available from ProQuest Dissertations \& Theses A\&I. (1664612134). Retrieved from http://search.proquest.com/docview/1664612134?accountid=12104

Focus Groups Advantages and Limitations Frontend User Experience Design Consultant. Retrieved February 17, 2012, from http://www.frontend.com/design/focus-groupsadvantages-limitations.html

Fowler, F. C., Chubb, J. E., \& Moe, T. M. (1991). The shocking ideological integrity of Chubb and Moe.

Freedman, S. W., \& Appleman, D. (2009). "In It for the Long Haul” How Teacher Education Can Contribute to Teacher Retention in High-Poverty, Urban Schools. Journal of Teacher Education, 60(3), 323-337. 
Fullan, M. (Ed.). (2003). The moral imperative of school leadership. Corwin press.

Fullan, M. (2009). Large-scale reform comes of age. Journal of educational change, 10(2-3), $101-113$.

Gibbs, A. (1997). Focus groups. Social research update, 19(8), 1-8.

Gillespie, N. A., \& Mann, L. (2004). Transformational leadership and shared values: The building blocks of trust. Journal of Managerial Psychology, 19(6), 588-607. Retrieved from http://search.proquest.com/docview/215868010?accountid=12104

Gulliksen, M. S., \& Hjardemaal, F. R. (2016). Choosing Content and Methods: Focus Group Interviews with Faculty Teachers in Norwegian Pre-Service Subject Teacher Education in Design, Art, and Crafts. Scandinavian Journal of Educational Research, 60(1), 1-19.

http://www.tcumn.edu/ rkrueger/focus.html.

Hurlburt, S., Le Floch, K. C., Therriault, S. B., Cole, S., \& National Center for Education Evaluation and Regional Assistance, (. (2011). Baseline Analyses of SIG Applications and SIG-Eligible and SIG-Awarded Schools. NCEE 2011-4019. National Center for Education Evaluation and Regional Assistance.

Husbands, S., Jowett, S., Barton, P., \& Coast, J. (2017). How Qualitative Methods Can be Used to Inform Model Development. PharmacoEconomics, 1-6.

Ingersoll, R. M. (2003). Who controls teachers' work? 
Ingersoll, R., Merrill, L., \& Stuckey, D. (2014). Seven trends: The transformation of the teaching force.

Ingersoll, Richard, and Henry May. "Recruitment, retention and the minority teacher shortage." (2011).

Jacob, B. A. (2007). The Challenges of Staffing Urban Schools with Effective Teachers. Future of Children, 17(1), 129-153.

Kaiser, K. (2009). Protecting respondent confidentiality in qualitative research. Qualitative Health Research, 19(11), 1632-1641.

Koh, W. L., Steers, R. M., \& Terborg, J. R. (1995). The effects of transformational leadership on teacher attitudes and student performance in Singapore. Journal of Organizational Behavior, 16(4), 319-333.

Krueger, R. A., \& Casey, M. A. (2000). A practical guide for applied research. A practical guide for applied research.

Krueger, R. A., \& Casey, M. A. (2002). Designing and conducting focus group interviews. Social Analysis, Selected Tools and Techniques, 4-23.

Krueger, R. A., \& Casey, M. A. (2014). Focus groups: A practical guide for applied research. Sage publications.

Kruger, R. (2003). Focus group interviewing. Retrieved January 19, 2012, from 
Kruger, R., \& Casey, M. A. (2000). Focus groups: A practical guide for applied research. Layton, L. (2015). Majority of US public school students are in poverty. Washington Post, January, 16 .

Leithwood, K., \& Jantzi, D. (1990). Transformational leadership: How principals can help reform school cultures. School effectiveness and school improvement, 1(4), 249-280.

Lewis, R., Finn, M. E., \& Johnson, L. (2005). Urban Education with an Attitude. Albany: State University of New York Press.

Lindsey, D. B., Jungwirth, L. D., Pahl, J. V., \& Lindsey, R. B. (Eds.). (2009). Culturally proficient learning communities: Confronting inequities through collaborative curiosity. Corwin Press.

National Education Association. (2008). Rural education. Washington, DC: Author.

Newland, C. A. (1996). Transformational challenges in Central and Eastern Europe and schools of public administration. Public Administration Review, 56(4), 382. Retrieved from http://search.proquest.com/docview/197164608?accountid=12104

Oakes, J., Franke, M. L., Quartz, K. H., \& Rogers, J. (2002). Research for high-quality urban teaching: Defining it, developing it, assessing it. Journal of Teacher Education, 53(3), 228-235.

Okçu, V. (2014). Relation between Secondary School Administrators' Transformational and Transactional Leadership Style and Skills to Diversity Management in the School. Educational Sciences: Theory and Practice, 14(6), 2162-2174. 
Olsen, B., \& Anderson, L. (2007). Courses of Action A Qualitative Investigation Into Urban Teacher Retention and Career Development. Urban Education, 42(1), 5-29.

Rabiee, F. (2004). Focus-group interview and data analysis. Proceedings of the nutrition society, 63(04), 655-660.

Raywid, M. A. (1999). Current Literature on Small Schools. ERIC Digest.

Razfar, A. (2011). Action Research in Urban Schools: Empowerment, Transformation, and Challenges. Teacher Education Quarterly, 38(4), 25-44.

Robinson, N. (1999). The use of focus group methodology—with selected examples from sexual health research. Journal of advanced nursing, 29(4), 905-913.

Silverman, D. (Ed.). (2016). Qualitative research. Sage.

Tesfaw, T. A. (2014). The Relationship between Transformational Leadership and Job Satisfaction: The Case of Government Secondary School Teachers in Ethiopia. Educational Management Administration \& Leadership, 42(6), 903-918. Thousand Oaks, CA: Sage.

Tipu, S. A. (n.d). Transformational leadership in Pakistan: An examination of the relationship of transformational leadership to organizational culture and innovation propensity. vol. 18, Bates, P., Michigan Univ., A. O., \& and, O. (1991). Poverty and Schools. Equity Coalition for Race, Gender, and National Origin, 2(2), 
Tolich, M. (2004). Internal confidentiality: When confidentiality assurances fail relational informants. Qualitative Sociology, 27, 101-106.

Vaughn, S., Schumm, J. S., \& Sinagub, J. M. (1996). Focus group interviews in education and psychology. Sage.

Wilson, W. J. (1988). The Ghetto Underclass and the Social Transformation of the Inner City. Black Scholar, 19(3), 10-17.

Wilson, W. J. (2011). Being Poor, Black, and American: The Impact of Political, Economic, and Cultural Forces. American Educator, 35(1), 10-23,

Wilson, W., \& Aponte, R. (1985). Urban Poverty. Annual Review of Sociology, 11, 231-258. Retrieved from http://www.jstor.org/stable/2083293 


\section{Appendices}

\section{(Appendix A)}

\section{Multifactor Leadership Questionnaire (MLQ) Form 6S}

INSTRUCTIONS: This questionnaire provides a description of your leadership style. Twenty-one descriptive statements are listed below. Judge how frequently each statement fits you. The word others may mean your followers, clients, or group members.

\section{KEY}

0 - Not at all 1 - Once in a while $2=$ Sometimes $3=$ Fairly often $4=$ Frequently, if not always

1. I make others feel good to be around me. 01234

2. I express with a few simple words what we could and should do. .01234

3. I enable others to think about old problems in new ways. 01234

4. I help others develop themselves. 01234

5. I tell others what to do if they wan t to be rewarded for their work. 01234 6. I am satisfied when others meet agreed-upon standards. 01234

7. I am content to let others continue working in the same ways always 01234

8. Others have complete faith in me. 01234 9. I provide appealing images about what we can do. 01234 10. . I provide others with new ways of looking at puzzling things. .01234

11. . I let others know how I think they are doing. 01234 12. . I provide recognition/rewards when others reach their goals. 13. As long as things are working, I do not try to change anything. 01234 14. Whatever others want to do is OK with me 01234 15. Others are proud to be associated with me 01234 16. . I help others find meaning in their work. 01234 17. . I get others to rethink ideas that they had never questioned before. 01234 18. . I give personal attention to others who seem rejected 01234 .

19. . I call attention to what others can get for what they accomplish. 01234 20. . I tell others the standards they have to know to carry out their work 21 . I ask no more of others than what is absolutely essential. 01234

\section{SCORING}

The MLQ-6S measures your leadership on seven factors related to transformational leadership. Your score for each factor is determined by summing three specified items on the questionnaire. For example, to determine your score for factor 1, Idealized influence, sum your responses for items 1, 8, and 15. Complete this procedure for all seven factors. TOTAL Idealized influence (items 1, 8, and 15)____ Factor 1 Inspirational motivation (items 2,9 , and 16) Intellectual stimulation (items 3, 10, and 17) Individual consideration (items 4,11 , and 18) Factor 2 Factor 3 Factor 4 
Contingent reward (items 5, 12, and 19)

Management-by-exception (items 6,13, and 20) .

Laissez-faire leadership (items 7,14 , and 21) .

Score range: HIGH $=912$,

MODERATE $=\mathbf{5 8}$,

LOW $=04$

SOURCE: Reproduced by special permission of the publisher, MIND GARDEN, Inc., www.mindgarden.com from the Multifactor Leadership Questionnaire by Bernard M. Bass and Bruce J. Avolio. Copyright (C 1995, 2000, 2004 by Bernard M. Bass and Bruce J. Avolio.
Factor 6

Factor 7 


\section{(Appendix B)}

\section{University of Missouri at Columbia}

\section{Recruitment Statement for Research Participation}

1. Chauncey E. Granger, Principal SEMS, Principal Investigator is inviting you to participate in this research study.

2. The title of this study is Transformational Leadership in Urban Educational Settings. My name is Chauncey E. Granger and I am interested in studying how the leadership/management styles of district officials are transforming underachieving, urban schools. This research will show how leaders are incorporating all stakeholders collaboratively to change the overall culture of schools that would create an atmosphere conducive to the real world of urban education and its many challenges. My rationale for choosing this topic is that there are many independent variables that are not factored in with the preparation process of being an educator in an urban school such as the neglect of the basic physical and social needs of students, deficit attitudes towards parents and students, as well as generational poverty and the many challenges that it may include such as, violence, drugs, the lack of access to healthy food options, health care, internal and external political influences, and many other challenges not mentioned that serve as roadblocks to a high quality education that is essential to our children's success.

It is my perception that there is a significant potential for this study to affect the broader educational community such as underperforming districts meeting state, (Missouri School Improvement Plan) and national requirements, (Every Student Succeeds Act).

3. Your willingness to participate would only require about one hour and thirty minutes to conduct an audio-recorded focus group interview/individual interview during the period of April 17, 2017, through April 21, 2017. In respect for each other, we ask that only one individual speak at one time in the group and that responses made by all participants be kept confidential. Your participation is voluntary and there is no penalty if you do not participate. If you are willing to participate in this study, please sign and return the enclosed informed consent form in the selfaddressed, stamped envelope. A response by April 14, 2017, is greatly appreciated. Once I receive your response, I will follow up to arrange a focus group interview/individual interview. If you are unable to participate in this study, an e-mail response declining participation would also be appreciated. Regardless of whether you choose to participate, please let me know if you would like a summary of my findings.

4. There are no risks involved in the participation in this study because no personally identifiable information will be collected, you do not have to answer questions that make you feel uncomfortable.

5. The results of this study may be published in educational research journals or presented at professional conferences. However, your name and identity will not be revealed and your record will remain anonymous. The interview information is Anonymous/de-identified: data contains no, identifiers, including code numbers that investigators can link to individuals. 
6. Participation in this study will not benefit you directly. However, you will have the opportunity to think introspectively and reflect on your own professional practice in ways you may not have considered before. Your participation may benefit others by having the opportunity to explore how their peers prepared for their leadership roles and use said information to advance their own professional growth and practice. Additionally, the end result would potentially be more informed school principals and school districts in how they support teachers to promote high quality mathematics instruction for all students regardless of their background and socioeconomic status.

7. You can choose not to participate. If you decide not to participate, there will not be a penalty to you or loss of any benefits to which you are otherwise entitled. You may withdraw from this study at any time. 


\section{(Appendix C)}

Focus Group Questions (School Leaders)

How would you describe the adult culture in your building and why?

How is buy in obtained with staff?

Is there a sense of family or a supportive team throughout our building?

Is risk taking encouraged at your school? If so how?

What is your evidence to back this claim?

What is the leadership's primary academic expectations for your school?

How do you know? / What does this look like?

How are academic expectations communicated?

What do PLCs look like in your team?

What systems are in place to support this initiative?

Are PLCs viable parts of a teacher's day in your school?

How has leadership protected time for teachers to participate in PLCs?

How does communication flow?

Is there any professional development to support areas of concerns in regards to teacher support in this process?

What does professional development look like at the building level?

How would you describe your principal's leadership style?

Is there anything that you would like to add? 


\section{(Appendix D)}

\section{Focus Group Questions}

How would you describe the adult culture in your building and why?

Is risk taking encouraged at your school? If so how?

Is there a sense of family or a supportive team throughout our building?

What is your evidence to back this claim?

What is the leadership's primary academic expectations for your school?

How so you know? / What does this look like?

How are academic expectations communicated?

What do PLCs look like in your team?

What systems are in place to support this initiative?

Are PLCs viable parts of a teacher's day in your school?

How has leadership protected time for teachers to participate in PLCs?

Is there any professional development to support areas of concerns in regards to teacher support in this process?

Is there anything that you would like to add? 


\section{(Appendix E)}

\section{Invitation to Participate}

March 14, 2017

Name:

Address

Dear Respondent,

My name is Chauncey E. Granger and I am interested in studying how the

leadership/management styles of district officials are transforming underachieving, urban

schools. This research will show how leaders are incorporating all stakeholders collaboratively to

change the overall culture of schools that would create an atmosphere conducive to the real

world of urban education and its many challenges. My rationale for choosing this topic is that

there are many independent variables that are not factored in with the preparation process of

being an educator in an urban school such as the neglect of the basic physical and social needs of students, deficit attitudes towards parents and students, as well as generational poverty and the many challenges that it may include such as, violence, drugs, the lack of access to healthy food options, health care, internal and external political influences, and many other challenges not mentioned that serve as roadblocks to a high quality education that is essential to our children's success.

It is my perception that there is a significant potential for this study to affect the broader educational community such as underperforming districts meeting state, (Missouri School Improvement Plan) and national requirements, (Every Student Succeeds Act). 
Your willingness to participate would only require about one hour and thirty minutes to conduct an audio-recorded focus group interview/individual interview during the period of April 17, 2017, through April 21, 2017. Your participation is voluntary and there is no penalty if you do not participate. If you are willing to participate in this study, please sign and return the enclosed informed consent form in the self-addressed, stamped envelope. A response by April 14, 2017, is greatly appreciated. Once I receive your response, I will follow up to arrange a focus group interview/individual interview. If you are unable to participate in this study, an e-mail response declining participation would also be appreciated. Regardless of whether you choose to participate, please let me know if you would like a summary of my findings.

If you have any questions or concerns about completing the questionnaire or about being in this study, you may contact me at (618) 741-1112 or my faculty advisor at the University of Missouri at Columbia, Department of Educational Leadership and Policy Analysis, 202 Hill Hall, Columbia, MO 65203. You may also contact the MU Campus Institutional Review Board at 483 McReynolds Hall, Columbia, MO 65211, (573) 882-9585, umresearchirb@missouri.edu if you have questions regarding your rights as a participant. The Institutional Review Board (IRB) at the University of Missouri at Columbia has approved this study.

Thank you for your consideration!

Sincerely,

Chauncey E. Granger

Doctoral Student

Educational Leadership and Policy Analysis

University of Missouri-Columbia 


\section{Please Detach This Paper and Return IT to the Project Researcher}

Please check the appropriate line to indicate that you have read and understand this letter:

I give consent to participate in this project designed to show how leaders are

incorporating all stakeholders collaboratively to change the overall culture of schools that would create an atmosphere conducive to the real world of urban education and its many challenges during the 2016-2017 school year.

One individual interview

One focus group interview

I would like more information before giving consent. Please call me at ( ） -

I do not give consent to participate.

Please bring a signed copy of the informed consent form to your interview and give it to the project researcher prior to the start of the interview, or if you need additional information prior to agreeing to participate please e-mail me at Eldridge.ceg@gmail.com or contact me at (618) 7411112.

Printed Name (first, middle initial, last)

Signed:

Date:

A copy of this form will be made and provided to you.

Please keep a copy for your records. 


\section{VITA}

Chauncey Granger has worked in the educational field for over 20 years. He has served in many capacities in the educational realm including truancy officer, teacher assistant, substitute teacher, special education teacher, assistant principal, and principal. He has a bachelor degree in speech communication, a master's degree in special education, a masters in administration k-12, and an educational specialist degree. He is currently completing his doctoral program at the University of Missouri at Columbia. He serves as Principal at Hazelwood Southeast Middle School, where he is in his sixth year in that role.

Chauncey is an adjunct professor at Lindenwood University in Hazelwood, Missouri at the north county location. At Lindenwood, he teaches evening masters level classes in elementary and secondary administration and also serves as a mentor to many of his students. Chauncey's career has primarily been in large urban centers such as Chicago Public Schools, St. Louis public Schools, The Hazelwood School District, and the Regional Office of Education in Belleville, Illinois where he served as a truant officer for the East St. Louis School District and Cahokia School District. "My mission and passion is to serve students and families in urban and impoverished areas similar to the one I grew up in and received a high quality education in." 